\title{
A High-Order Discontinuous Galerkin Solver for Helically Symmetric Flows
}

\author{
Dominik Dierkes $^{1, *}$, Florian Kummer ${ }^{1}$ and Dominik Plümacher ${ }^{1}$ \\ ${ }^{1}$ Department of Mechanical Engineering, Technische Universität Darmstadt, \\ Otto-Berndt-Straße 2, 64287 Darmstadt, Germany.
}

Received 21 November 2019; Accepted (in revised version) 12 January 2021

\begin{abstract}
We present a high-order discontinuous Galerkin (DG) scheme to solve the system of helically symmetric Navier-Stokes equations which are discussed in [28]. In particular, we discretize the helically reduced Navier-Stokes equations emerging from a reduction of the independent variables such that the remaining variables are: $t, r, \xi$ with $\xi=a z+b \varphi$, where $r, \varphi, z$ are common cylindrical coordinates and $t$ the time. Beside this, all three velocity components are kept non-zero. A new non-singular coordinate $\eta$ is introduced which ensures that a mapping of helical solutions into the three-dimensional space is well defined. Using that, periodicity conditions for the helical frame as well as uniqueness conditions at the centerline axis $r=0$ are derived. In the sector near the axis of the computational domain a change of the polynomial basis is implemented such that all physical quantities are uniquely defined at the centerline.

For the temporal integration, we present a semi-explicit scheme of third order where the full spatial operator is splitted into a Stokes operator which is discretized implicitly and an operator for the nonlinear terms which is treated explicitly. Computations are conducted for a cylindrical shell, excluding the centerline axis, and for the full cylindrical domain, where the centerline is included. In all cases we obtain the convergence rates of order $\mathcal{O}\left(h^{k+1}\right)$ that are expected from DG theory.

In addition to the first DG discretization of the system of helically invariant NavierStokes equations, the treatment of the central axis, the resulting reduction of the DG space, and the simultaneous use of a semi-explicit time stepper are of particular novelty.
\end{abstract}

AMS subject classifications: 76T99, 35J75

Key words: Discontinuous Galerkin (DG), helical flows.

*Corresponding author. Email addresses: dierkes@fdy.tu-darmstadt.de (D. Dierkes),

kummer@fdy.tu-darmstadt.de (F. Kummer), pluemacher@fdy.tu-darmstadt.de (D. Plümacher) 


\section{Introduction}

Helical flow structures appear in various natural phenomena and technological devices, for example, in the wake of windmills [39], as wing tip vortices [32], in astrophysical plasmas (see e.g. [4]) and in laboratory applications, including "straight tokamak" plasma flow approximations, (see e.g. [27,35]) and other experiments. In particular, helical vortex structures were observed by [34] in experiments with swirling flows in a cylindrical tube, and as such, they are part of the various flow structures observed in the known vortex breakdown.

Various groups have worked on the theoretical description of helical flows in recent decades. The simplest approach here is to introduce a helical coordinate $\xi=a z+b \varphi$, $a, b=$ const. $\neq 0$ and to assume that all physical quantities depend on the cylinder radius $r$ and the helical coordinate $\xi$. Helically invariant flows include translationally and axially invariant ones as special cases. For both steady Euler equations describing incompressible fluid flows and for plasma equilibrium equations in the magnetohydrodynamics (MHD) framework, the helical invariance requirement allows to reduce the governing equations to a single partial differential equation (PDE) known as the JFKO equation [27]. This important equation generalizes the famous Bragg-Hawthorne-GradRubin-Shafranov equation $[7,23,36]$ describing steady axisymmetric inviscid flows onto the helically invariant case. Families of exact solutions of JFKO equations are known, including those derived by [6] (see also [5,10]). In the more general context of helical geometry, several works focused on twisted pipes following a given spatial curve (see $[19,20,38,41])$. Using non-orthogonal and local-orthogonal coordinate systems, the effects of pipe curvature and torsion on the flow were investigated. Special analytical solutions of steady flows in helically symmetric pipes were found by [43]. In [12] a DNS code for the helical invariant Navier-Stokes equations in a generalized vorticitystreamfunction formulation has been developed. In [16] the three-dimensional Euler equations are reduced to a linear equation, assuming that the flow has helical symmetry and consists of a rigidly rotating basic part and a Beltrami disturbance part. Further, the authors derived exact solutions for flows in a straight pipe of circular cross section. Exact solutions for helical flows of a Maxwell fluid constrained between two infinite coaxial circular cylinders were derived by [26]. The present introduction as well as additional results on helical flows can be found in [14].

The full three dimensional system of incompressible constant-density Euler- and Navier-Stokes equations under the assumption of helical symmetry have been derived and analyzed in [28]. In particular, various new conservation laws admitted by the model have been found in primitive variables and using the vorticity formulation. A general helically symmetric setting was used, where all three velocity components and the pressure are generally nonzero, and may depend on the time $t$, the cylindrical radius $r$, and the helical variable

$$
\xi=a z+b \varphi
$$


In a general helically symmetric setting, no restrictive assumptions are made concerning the form of the velocity components or the pressure. The consideration is based exclusively on the independence from the third spatial variable which changes along each helix $r=$ const., $\xi=$ const.. Thus the flow has two spatial dimensions, and is called $(2+1)$ dimensional in space-time. Since the spatial dimensions are reduced to two, but all three components of the velocity vector are nonzero, helical flows are often referred to as " $2 \frac{1}{2}$ dimensional". In turbulence research, a flow is denoted as a two-component flow if one of the velocity components vanishes.

Helical invariance of the Navier-Stokes equations is a consequence of its admitted Lie group of point symmetries, specifically, the invariance of the model with respect to rotations and translations about the $z$-axis. The helical invariance thus generalizes and includes the axial symmetry (achieved at $a=1, b=0$ ) and the $z$-translation symmetry (corresponding to $a=0, b=1$ ). In 2017, Dierkes and Oberlack extended the work [28] by introducing a more general, time-dependent helical coordinate system, based on rotation and Galilei invariance of the Navier-Stokes model. The new approach used a time-dependent helical variable $\xi=z / \alpha(t)+b \varphi$, with $b=$ const., and $\alpha(t)$ is an arbitrary function of time $t$. This coordinate system describes helical flows with a time-dependent helical pitch; both Euler and Navier-Stokes equations admit a reduced invariant form with respect to this extended helical coordinate system.

During the last decades, the discontinuous Galerkin (DG) method has been used to solve the Stokes- and the incompressible Navier-Stokes equations in various works. Girault et al. derived an inf-sup condition for a velocity of degree $k$ and a pressure of degree $k-1$, for $1 \leq k \leq 3$ in two dimensions, for grids with hanging nodes and triangular elements [21]. Later they extended these results to instationary flows [22]. The stability of equal-order discretizations for velocity and pressure requires an additional stabilization term in the continuity equation, but can be shown for more general settings in 2D and 3D with arbitrary polynomial orders, cf. the textbook of di Pietro and Ern [13].

For unsteady two-dimensional flows, a DG solver has been developed by Ferrer and Willden [18], whereas in three dimensions a DG solver is presented in [37], in which a semi explicit temporal discretization with explicit treatment of the nonlinear term and implicit treatment of the Stokes operator is used. They both use the Interior Penalty (IP) Galerkin formulation [1]. Recent development of the DG method for steady and unsteady Navier-Stokes equations on arbitrary grids is proposed in [44], where a simplified artificial compressibility is used to discretize the inviscid term. The authors further discretized the viscous term by the direct DG (DDG) method. An innovative possibility to combine a high-order finite difference (FD) scheme with DG is shown in the work of [42], where the DG method is used to treat the boundary conditions while a novel high-order FD scheme is applied to simulate the flow. New developments of the DG method are proposed in [9] where an adjoint-based h-adaptive high-order reconstructed DG method is introduced and used to solve the two-dimensional steady-state compressible Euler equations.

In addition to that, an extended DG method has been developed for solving multi- 
phase problems [30]. The DG method has also been applied to PDE's which are written in non-cartesian coordinates, e.g. in [33] the shallow water equations are discretized on a cubic sphere. There are also numerous works concerning a numerical discretization of the incompressible Navier-Stokes equations in polar and cylindrical coordinates, most applying spectral elements [31] and finite difference schemes [3, 24, 40] for the spatial discretization. They all have in common that due to the singularities in the equations a special treatment at the centerline axis, where the radial coordinate $r$ is zero needs to be taken into account. For instance, in [31] so-called essential and natural pole conditions are discussed, which are necessary for the well-posedness and the regularity of the solutions at the centerline, respectively. In [11] governing equations for the flow at the centerline are derived using series expansions near $r=0$, whereas in [29] the authors demand smoothness of all physical variables along the centerline which results in constraints for the velocity and the pressure.

In the current paper, we will present a DG formulation of the helically invariant Navier-Stokes equations. This can also be seen as a discontinuous Petrov-Galerkin (DPG) formulation since all equations are multiplied by a metric function in order to ensure well-posedness, smoothness and regularity of the numerical solutions along the centerline. An example of the development of a DPG method for different convection-diffusion type problems may be found in [17].

In addition to that, we derive centerline conditions at the axis $r=0$, combining the periodicity condition in the helical setting (cf. Appendix B) and the condition for uniqueness at the centerline, derived in Appendix C. The final centerline conditions presented in Section 5 are realized using a reduced DG basis which is shown in Section 5.1. The article is heavily based on a part of [14] which is the author's PhD thesis. Since until today there is no numerical discretization of the system of helically invariant Navier-Stokes equations, the following contents of this publication are of particular importance for the numerical investigation of helically symmetric flows: the derivation of the weak form using the Petrov-Galerkin method, the formulation and implementation of suitable periodicity and smoothness conditions at the centerline axis $r=0$ resulting in a reduction of the DG space, and the use of a semi-explicit time stepper to solve the unsteady system of PDEs.

The paper is organized as follows: In Section 2 the continuous setting of the helically invariant Navier-Stokes equations is introduced and basic definitions are presented. Section 3 focuses on the numerical discretization of the PDE system including the definition of the reduced DG space as well as on the temporal discretization using a semi-explicit time-stepping method. The Sections 4 and 5 show the results through a discussion of convergence studies for simulations on a cylindrical shell and on the full cylindrical domain. The latter require the centerline conditions which are also presented and applied in Section 5. In Section 6 the conclusion and an outlook for manifold applications of the developed code is given. 


\section{Helically invariant Navier-Stokes equations (the continuous setting)}

The helical coordinate system $(r, \eta, \xi)$ has been introduced originally in [28]. It is given by

$$
r, \quad \xi=a z+b \varphi, \quad \eta=a \varphi-b z / r^{2},
$$

where $a, b=$ const., $a^{2}+b^{2}>0$ and $(r, \varphi, z)$ are the usual cylindrical coordinates. In the present work we use a new invariant coordinate $\eta$, given by

$$
\eta=-b z+a r^{2} \varphi
$$

which has two important advantages. First, the coordinate lines of $\eta$ (lines where $\eta=$ const.) are orthogonal to lines where $\xi=$ const. and second, the coordinate $\eta$ is not singular at the centerline $r=0$, i.e. $\eta$ does not collapse to one single point at the origin $z=0, r=0$. However, the choice of the invariant coordinate $\eta$ is important for the formulation of appropriate conditions at the centerline of the helix $r=0$, which we consider in Section 5 . The derivation of the coordinate (2.2) is provided in Appendix A.

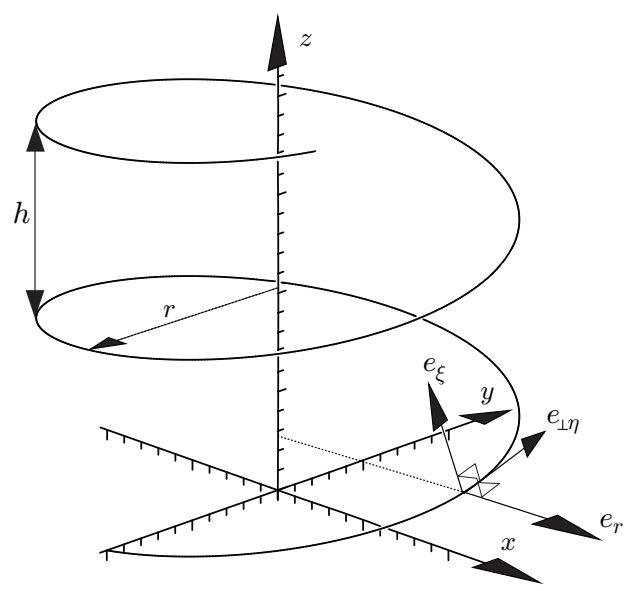

Figure 1: An illustration of the helix $\xi=$ const. for $a=1, b=-h / 2 \pi$, where $h$ is the $z$-step over one helical turn. Basis unit vectors in the helical coordinates.

On each cylinder $r=$ const., the lines $\xi=$ const. and $\eta=$ const. correspond to two families of helices which are orthogonal to each other. By choosing the constants $a, b$ one obtains a specific helical frame. In the limiting case, if $a=1, b=0$, the helical coordinates become cylindrical coordinates with $\eta=r^{2} \varphi, \xi=z$. Importantly, the curvilinear helical coordinates $(r, \eta, \xi)$ do not form an orthogonal triple. Although the unit direction vectors of the coordinates $(r, \xi)$ are orthogonal, it can be shown that there is no third coordinate that is orthogonal to both $r$ and $\xi$ and that can be consistently introduced in any open 
ball $B \in \mathbb{R}^{3}$. A locally orthogonal triple of unit vectors

$$
\boldsymbol{e}_{r}=\frac{\nabla r}{|\nabla r|}, \quad \boldsymbol{e}_{\xi}=\frac{\nabla \xi}{|\nabla \xi|}, \quad \boldsymbol{e}_{\perp \eta}=\frac{\nabla_{\perp} \eta}{\left|\nabla_{\perp} \eta\right|}=\boldsymbol{e}_{\xi} \times \boldsymbol{e}_{r}
$$

is defined at every point for the purpose of expansion of vector quantities in a natural basis.

We define a helically invariant function as a function which is independent of $\eta$, and has the form $F(t, r, \xi)$. We will assume that all physical variables are $\eta$-independent such that we obtain exact solutions for helically invariant flows. Throughout the paper, upper indices will refer to the corresponding components of vector fields (vorticity, velocity, etc.), and lower indices will denote partial derivatives. For example,

$$
u_{\xi}^{\eta} \equiv \frac{\partial}{\partial \xi} u^{\eta}(t, r, \xi)
$$

We also assume summation in all repeated indices.

In nabla-based notation in Cartesian coordinates, the Navier-Stokes equations of incompressible viscous fluid flows without external forces are given by

$$
\begin{aligned}
& \nabla \cdot \boldsymbol{u}=0, \\
& \boldsymbol{u}_{t}+(\boldsymbol{u} \cdot \nabla) \boldsymbol{u}+\nabla p-v \nabla^{2} \boldsymbol{u}=0,
\end{aligned}
$$

where the fluid velocity vector $\boldsymbol{u}=u^{1} \boldsymbol{e}_{x}+u^{2} \boldsymbol{e}_{y}+u^{3} \boldsymbol{e}_{z}$ and fluid pressure $p$, in which the density has already been absorbed, are functions of $x=(x, y, z)$ and $t$. The viscosity coefficient $v=$ const.; the inviscid case $v=0$ yields the Euler equations.

In order to rewrite the equations (2.4) in a helically symmetric setting, one may write the velocity vector in the cylindrical and the helical basis:

$$
\boldsymbol{u}=u^{r} \boldsymbol{e}_{r}+u^{\varphi} \boldsymbol{e}_{\varphi}+u^{z} \boldsymbol{e}_{z}=u^{r} \boldsymbol{e}_{r}+u^{\eta} \boldsymbol{e}_{\perp \eta}+u^{\xi} \boldsymbol{e}_{\xi},
$$

where $u^{r}, u^{\varphi}, u^{z}$ are the velocity components in cylindrical coordinates. The helical velocity components are related to the cylindrical velocity components by

$$
u^{\eta}=\boldsymbol{u} \cdot \boldsymbol{e}_{\perp \eta}=B\left(a u^{\varphi}-\frac{b}{r} u^{z}\right), \quad u^{\xi}=\boldsymbol{u} \cdot \boldsymbol{e}_{\xi}=B\left(\frac{b}{r} u^{\varphi}+a u^{z}\right),
$$

and backward,

$$
u^{\varphi}=B\left(a u^{\eta}+\frac{b}{r} u^{\xi}\right), \quad u^{z}=B\left(-\frac{b}{r} u^{\eta}+a u^{\xi}\right)
$$

where

$$
B(r)=\frac{r}{\sqrt{a^{2} r^{2}+b^{2}}}
$$


In what follows, for brevity, we will often write $B(r)=B$ and $d B(r) / d r=B^{\prime}$.

By transforming the PDEs to helical coordinates, and assuming helical invariance $\partial / \partial \eta \equiv 0$ for all velocity components and the pressure, one obtains the continuity equation and the three components of the vector momentum equation, which represent the helically invariant Navier-Stokes system in primitive variables:

$$
\begin{aligned}
& \frac{1}{r} u^{r}+u_{r}^{r}+\frac{1}{B} u_{\xi}^{\xi}=0, \\
& u_{t}^{r}+u^{r} u_{r}^{r}+\frac{1}{B} u^{\xi} u_{\xi}^{r}-\frac{B^{2}}{r}\left(\frac{b}{r} u^{\xi}+a u^{\eta}\right)^{2} \\
& =-p_{r}+v\left[\frac{1}{r}\left(r u_{r}^{r}\right)_{r}+\frac{1}{B^{2}} u_{\xi \xi}^{r}-\frac{1}{r^{2}} u^{r}-\frac{2 b B}{r^{2}}\left(a u_{\xi}^{\eta}+\frac{b}{r} u_{\xi}^{\xi}\right)\right], \\
& u_{t}^{\eta}+u^{r} u_{r}^{\eta}+\frac{1}{B} u^{\xi} u_{\xi}^{\eta}+\frac{a^{2} B^{2}}{r} u^{r} u^{\eta} \\
& =v\left[\frac{1}{r}\left(r u_{r}^{\eta}\right)_{r}+\frac{1}{B^{2}} u_{\xi \xi}^{\eta}+\frac{a^{2} B^{2}\left(a^{2} B^{2}-2\right)}{r^{2}} u^{\eta}+\frac{2 a b B}{r^{2}}\left(u_{\xi}^{r}-\left(B u^{\xi}\right)_{r}\right)\right], \\
& u_{t}^{\xi}+u^{r} u_{r}^{\xi}+\frac{1}{B} u^{\xi} u_{\xi}^{\xi}+\frac{2 a b B^{2}}{r^{2}} u^{r} u^{\eta}+\frac{b^{2} B^{2}}{r^{3}} u^{r} u^{\xi} \\
& =-\frac{1}{B} p_{\xi}+v\left[\frac{1}{r}\left(r u_{r}^{\xi}\right)_{r}+\frac{1}{B^{2}} u_{\xi \xi}^{\xi}+\frac{a^{4} B^{4}-1}{r^{2}} u^{\xi}+\frac{2 b B}{r}\left(\frac{b}{r^{2}} u_{\xi}^{r}+\left(\frac{a B}{r} u^{\eta}\right)_{r}\right)\right],
\end{aligned}
$$

where the velocity components $u^{r}, u^{\eta}, u^{\xi}$ and the pressure $p$ are functions of $r, \xi$ and $t$ and the geometric factor $B$ is given by (2.8). Due to the $2 \pi$-periodicity of the cylindrical polar angle $\varphi$, in order to be globally defined, every component of a helically invariant solution must be periodic in $\xi$ with the period

$$
\tau_{\xi}=2 \pi b
$$

The helically invariant reduction (2.9) of the Navier-Stokes equations has been extensively investigated in Kelbin et al., where various new conservation laws, including families of conservation laws, have been found for the viscous $(v \neq 0)$ and the inviscid $(v=0)$ case. As an example, for the inviscid case, conservation laws of kinetic energy and $z$-projections of momentum and angular momentum have been discovered, as well as a new infinite family of conserved generalized momenta/angular momenta. For the viscous case, a z-projection of momentum and an additional momentum-like quantity $(r / B) u^{\eta}$ are conserved.

\section{The discontinuous Galerkin discretization of the helically invariant Navier-Stokes equations}

In this section we present the numerical discretization of the PDE system (2.9). After some basic definitions in Section 3.1 the numerical fluxes of the spatial discretization 
are presented in Section 3.2 and a semi-explicit scheme for the temporal discretization is discussed in Section 3.3. Subsequently, the computational domain will be restricted to a cylindrical shell, i.e. the centerline axis is excluded and the radial coordinate will be $R_{0} \leq r \leq R_{1}$, where $R_{0} \geq 0$. Afterwards, the full computational domain, including the centerline axis at $r=0$ will be included. For each computational domain spatial and temporal convergence studies are conducted for a given test case discussed in Section 4.1 .

\subsection{Definitions}

In order to keep the readability as simple as possible, we introduce some standard definitions which we use for the DG discretization, cf. [30]. These are all well known and may be found in similar form in common textbooks like [13,25].

Definition 3.1 (basic notations). We define:

- the domain: $\Omega=(0, \cdots, 1) \times(0, \cdots, 2 \pi)$ is in all cases $2 \pi$-periodic (cf. Appendix B);

- the numerical grid: $\mathfrak{K}_{h}=\left\{K_{1}, \cdots, K_{J}\right\}$, with $h$ being the shorter edge of the rectangular cell;

- the set containing all edges of the grid: $\Gamma:=\bigcup_{j} \partial K_{j}$. Furthermore, the set of all internal edges: $\Gamma_{\text {int }}:=\Gamma \backslash \partial \Omega$;

- a normal field $n_{\Gamma}$ on $\Gamma$. On $\partial \Omega$, it represents an outer normal, i.e., on $\partial \Omega, n_{\Gamma}=n_{\partial \Omega}$;

- At the mesh skeleton, the inner- resp. outer-value of a field $u \in \mathcal{C}^{0}\left(\Omega \backslash \Gamma_{\text {int }}\right)$ are defined as:

$$
\begin{aligned}
& u^{i n}(\boldsymbol{x}):=\lim _{\epsilon \searrow 0} u\left(\boldsymbol{x}-\epsilon \boldsymbol{n}_{\Gamma}\right) \text { for } \boldsymbol{x} \in \Gamma, \\
& u^{\text {out }}(\boldsymbol{x}):=\lim _{\epsilon \searrow 0} u\left(\boldsymbol{x}+\epsilon \boldsymbol{n}_{\Gamma}\right) \text { for } \boldsymbol{x} \in \Gamma_{\mathrm{int}} .
\end{aligned}
$$

Then, the jump and average value operator are defined as

$$
\begin{aligned}
& {[[u]]:= \begin{cases}u^{\text {in }}-u^{\text {out }} & \text { on } \Gamma_{\text {int }}, \\
u^{\text {in }} & \text { on } \partial \Omega,\end{cases} } \\
& \{u\}:= \begin{cases}\left(u^{\text {in }}+u^{\text {out }}\right) / 2 & \text { on } \Gamma_{\text {int }}, \\
u^{\text {in }} & \text { on } \partial \Omega ;\end{cases}
\end{aligned}
$$

- the broken polynomial space of total degree $k$ :

$$
\mathbb{P}_{k}\left(\mathfrak{K}_{h}\right):=\left\{f \in L^{2}(\Omega) ; \forall K \in \mathfrak{K}_{h}:\left.f\right|_{K} \text { is polynomial and } \operatorname{deg}\left(\left.f\right|_{K}\right) \leq k\right\} ;
$$


- the broken gradient $\nabla_{h}$ : for $u \in \mathcal{C}^{1}(\Omega \backslash \Gamma), \nabla_{h} u$ denotes the gradient on the domain $\Omega \backslash \Gamma$; in analog fashion, the broken divergence $\operatorname{div}_{h}(\boldsymbol{u})$;

- the standard-basis vector $\boldsymbol{e}_{d}$, for $d \in\{1,2\}: \boldsymbol{e}_{1}=\boldsymbol{e}_{r}=(1,0), \boldsymbol{e}_{2}=\boldsymbol{e}_{\xi}=(0,1)$;

- the metric function $f: \mathbb{R} \rightarrow \mathbb{R} ; r \mapsto f(r)$ for which we choose $f(r)=(B(r))^{2}$, where $B(r)$ is the geometric function (2.8), naturally arising from the transformation into the helical system.

\subsection{DG spaces and the spatial discretization}

In the following, we first introduce DG spaces, which ensure the conditions at the centerline axis $r=0$ given by

$$
u^{r}=0, \quad u^{\xi}=0, \quad u_{\xi}^{\eta}=0, \quad p_{\xi}=0
$$

Further details about the centerline conditions are presented in Section 5 and a derivation can be found in the Appendices B and C. After the definition of the DG spaces we describe the spatial discretization of the helically invariant Navier-Stokes equations (2.9).

\subsubsection{Reduced DG spaces ensuring the centerline conditions}

In standard DG formulation, the velocity and pressure are discretized in DG spaces of order $k$ and $k^{\prime}=k-1$, respectively, in order to comply the Ladyženskaja-Babuška-Brezzi (LBB) condition $[2,8]$. In this case, the DG space is usually given by

$$
(\boldsymbol{u}, p) \in \mathbb{P}_{k}(\mathcal{K})^{3} \times \mathbb{P}_{k-1}(\mathcal{K}):=\mathbb{V}_{k} .
$$

In contrast to that, for the discretization helically invariant Navier-Stokes equations (2.9) we need to introduce a reduced DG space $\mathbb{V}_{k}^{0}$ due to the centerline conditions at the central axis $r=0$. We distinguish between two different spatial domains in the following. The first domain is a cylindrical shell where the centerline axis $r=0$ is omitted (domain \#1). Afterwards, we consider the full domain including the centerline axis which we denote as the second case (domain \#2). For both, we distinguish between a mixed-order and an equal-order formulation of the DG discretization. For the DG discretization on the cylindrical shell we seek solutions in the DG space $\mathbb{V}_{k}$ where we additionally demand that the integral of the pressure over one cell vanishes, i.e. $\int_{K_{0}} p=0$. Here, $K_{0}$ denotes the cell in the computational domain where the latter condition holds. For the DG discretization in the full domain, the underlying DG space is denoted as $\mathbb{V}_{k}^{0}$. The different DG spaces for the four distinct cases are presented in Table 1. The validation of the implementation in the cylindrical shell is presented in Section 4 followed by the validation in the full domain in Section 5.

For the DG discretization of the helically invariant Navier-Stokes equations (2.9), we use the following boundary conditions (BCs)

$$
\begin{array}{ll}
\boldsymbol{u}=\boldsymbol{u}_{D} & \text { on } \Gamma_{D}, \\
\boldsymbol{u}=\boldsymbol{u}_{P} & \text { on } \Gamma_{P},
\end{array}
$$


Table 1: DG spaces for different geometries and DG formulations. $\mathbb{P}_{k}(\mathcal{K})$ and $\mathbb{P}_{k-1}(\mathcal{K})$ are the spaces of smooth polynomial functions up to order $k$ and $k-1$, respectively.

\begin{tabular}{|c|c|c|}
\hline Geometry & mixed-order & equal-order \\
\hline 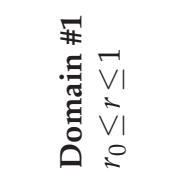 & $\begin{array}{c}\mathbb{V}_{k}^{0}=\left\{(\boldsymbol{u}, p) \in \mathbb{P}_{k}(\mathcal{K})^{3} \times \mathbb{P}_{k-1}(\mathcal{K}),\right. \\
\left.\text { s.t. } \int_{K_{0}} p=0\right\}\end{array}$ & $\begin{array}{c}\mathbb{V}_{k}^{0}=\left\{(\boldsymbol{u}, p) \in \mathbb{P}_{k}(\mathcal{K})^{3} \times \mathbb{P}_{k}(\mathcal{K}),\right. \\
\left.\text { s.t. } \int_{K_{0}} p=0\right\}\end{array}$ \\
\hline 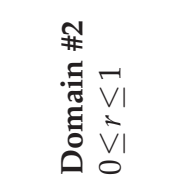 & $\begin{array}{r}\mathbb{V}_{k}^{0}=\left\{(\boldsymbol{u}, p) \in \mathbb{P}_{k}(\mathcal{K})^{3} \times \mathbb{P}_{k-1}(\mathcal{K}),\right. \\
\left.\text { s.t. } u^{r}=u^{\xi}=0, u_{\xi}^{\eta}=0, p_{\xi}=0\right\}\end{array}$ & $\begin{array}{r}\mathbb{V}_{k}^{0}=\left\{(\boldsymbol{u}, p) \in \mathbb{P}_{k}(\mathcal{K})^{3} \times \mathbb{P}_{k}(\mathcal{K}),\right. \\
\left.\text { s.t. } u^{r}=u^{\xi}=0, u_{\xi}^{\eta}=0, p_{\xi}=0\right\}\end{array}$ \\
\hline
\end{tabular}

where $\Gamma_{D}$ is the Dirichlet boundary and $\Gamma_{P}$ is the boundary where periodic BCs are assumed. For the Dirichlet $B C$ we distinguish between the two spatial domains (i) the cylindrical shell and (ii) the full domain including $r=0$. In the case (i) we assume Dirichlet $\mathrm{BC}$ s at the inner and the outer cylindrical walls, given by

$$
\Gamma_{D}=\left\{(r, \xi) \in \mathbb{R}^{2} \mid r=r_{0}>0 \vee r=1\right\},
$$

whereas in case (ii) Dirichlet BCs are assumed only at the outer cylindrical wall, i.e.

$$
\Gamma_{D}=\left\{(r, \xi) \in \mathbb{R}^{2} \mid r=1\right\} .
$$

At the inner axis $r=0$ the centerline conditions (3.4) are used. In both cases periodic boundary conditions are implemented on the following boundaries

$$
\Gamma_{P}=\left\{(r, \xi) \in \mathbb{R}^{2} \mid \xi=0 \vee \xi=2 \pi\right\} .
$$

\subsubsection{The spatial discretization of the helically invariant Navier-Stokes equations}

We propose the following discretization of the helically invariant Navier-Stokes equations (2.9), using the boundary conditions (3.6) in the DG space: find $(\boldsymbol{u}, p) \in \mathbb{V}_{k}^{0}$ such that for all $(v, \tau) \in \mathbb{V}_{k}^{0}$

$$
\operatorname{Ns}(\boldsymbol{u},(\boldsymbol{u}, p),(\boldsymbol{v}, \tau))=\operatorname{rhsNs}((\boldsymbol{v}, \tau)),
$$

where the Navier-Stokes form $\mathrm{Ns}(-,-,-)$ is given by

$$
\operatorname{Ns}(\boldsymbol{u},(\boldsymbol{u}, p),(\boldsymbol{v}, \tau))=\mathcal{N}(\boldsymbol{u}, \boldsymbol{u}, \boldsymbol{v})-\mathcal{A}(p, \boldsymbol{u}, \boldsymbol{v}),
$$

which consists of a trilinear form $\mathcal{N}(-,-,-)$ representing the convective terms, and a bilinear form $\mathcal{A}(p, u, v)$, representing the pressure gradient and the viscous terms of the 
Navier-Stokes equations (2.9). Note that (3.10) is the discrete spatial formulation of (2.9), neglecting the transient term, which will be taken into account in Section 3.3. The righthand side of the discretized Navier-Stokes equations (2.9),

$$
\operatorname{rhsNs}((\boldsymbol{v}, \tau))=s(\boldsymbol{v})+q(\boldsymbol{v})+r(\tau),
$$

is the sum of three linear forms, representing body forces by $s(v)$, boundary conditions for the momentum equations by $q(v)$ and boundary conditions for the continuity equation by $r(\tau)$.

The momentum equations of the helically invariant Navier-Stokes equations (2.9) are presented component-wise in radial direction as well as in the helical " $\xi$ " and " $\eta$ "direction. For brevity, we introduce the following naming for the DG space

$$
\mathbb{V}_{k, i}^{0}:=\left\{u_{i} \mid\left(u_{1}, u_{2}, u_{3}, u_{4}\right)=(\boldsymbol{u}, \tau) \in \mathbb{V}_{k}^{0}\right\}
$$

and using that, we write the system (3.10) component-wise as follows

$$
\begin{aligned}
\mathcal{C}(\boldsymbol{u}, \tau) & =0 & & \forall \tau \in \mathbb{V}_{k, 4}^{0}, \\
\mathcal{N}_{1}\left(\boldsymbol{u}, \boldsymbol{u}, v_{1}\right)-\mathcal{A}_{1}\left(p, \boldsymbol{u}, v_{1}\right) & =b_{1}\left(v_{1}\right) & & \forall v_{1} \in \mathbb{V}_{k, 1}^{0}, \\
\mathcal{N}_{2}\left(\boldsymbol{u}, \boldsymbol{u}, v_{2}\right)-\mathcal{A}_{2}\left(\boldsymbol{u}, v_{2}\right) & =b_{2}\left(v_{2}\right) & & \forall v_{2} \in \mathbb{V}_{k, 2}^{0}, \\
\mathcal{N}_{3}\left(\boldsymbol{u}, \boldsymbol{u}, v_{3}\right)-\mathcal{A}_{3}\left(p, \boldsymbol{u}, v_{3}\right) & =b_{3}\left(v_{3}\right) & & \forall v_{3} \in \mathbb{V}_{k, 3}^{0},
\end{aligned}
$$

where the index "1" denotes the terms of the momentum equation in radial direction, the index " 2 " and " 3 " represent terms of the $\eta$ - and $\xi$-momentum equation. Note that the $\eta$-momentum equation has no pressure. Furthermore, (3.14a) is the discrete form of the continuity equation.

In the following, we present the DG discretization of each term in (3.14). The velocity divergence (3.14a) is discretized as follows

$$
\begin{aligned}
\mathcal{C}(\boldsymbol{u}, \tau)= & -\int_{\Omega}\left[u^{r} \cdot\left(\frac{\mathrm{d} f}{\mathrm{~d}_{h} r} \tau+f \partial_{r, h} \tau\right)+\frac{f}{B} u^{\xi} \partial_{\xi, h} \tau-\frac{f}{r} u^{r} \tau\right] \mathrm{d} V \\
& +\oint_{\Gamma}\left[\left\{u^{r}\right\} \boldsymbol{e}_{r} \cdot \boldsymbol{n}+\frac{1}{B}\left\{u^{\xi}\right\} \boldsymbol{e}_{\xi} \cdot \boldsymbol{n}\right] f[[\tau]] \mathrm{d} S,
\end{aligned}
$$

where $f=f(r)$ is a metric function, which we introduce to remove singularities in the coefficients of the PDEs at the centerline axis $r=0$. In the present work we choose $f=B^{2}(r)$. The convective terms are discretized in a "super weak form", by partially integrating twice

$$
\mathcal{N}(w, u, v)=\int_{\Omega} \nabla u \cdot w f v \mathrm{~d} V+\oint_{\Gamma}(\widehat{u w n}-u w n) f v \mathrm{~d} S .
$$

The parameter $\boldsymbol{w}=\left(w_{0}^{r}, w_{0}^{\eta}, w_{0}^{\xi}\right)$ is taken from the initial condition and linearizes the convective part. The operator $\mathcal{N}$ is the sum of the convective terms of the component-wise 
discretization (3.14), i.e. $\mathcal{N}=\mathcal{N}_{1}+\mathcal{N}_{2}+\mathcal{N}_{3}$. The scalar quantity $u$ is the velocity component for each momentum equation, e.g. $u=u^{r}$ for the $r$-momentum equation. For the flux $\widehat{u w n}$, we use the upwind formulation,

$$
f_{u p w}(u, w, n):=\widehat{u w n}= \begin{cases}u^{-} w \cdot n & \text { if } w \cdot n \geq 0 \\ u^{+} w \cdot n & \text { if } w \cdot n<0\end{cases}
$$

For brevity, we introduce the flux

$$
f_{s w}(u, w, n):=f_{u p w}-u w n,
$$

such that the convective terms in (3.14) read

$$
\begin{aligned}
\mathcal{N}_{1}\left(\boldsymbol{u}, \boldsymbol{w}, v_{1}\right)= & \int_{\Omega}\left[u_{0}^{r} \partial_{r, h} u^{r}+\frac{1}{B} u_{0}^{\xi} \partial_{\xi, h} u^{r}-\frac{B^{2}}{r}\left(\frac{b}{r} u_{0}^{\xi}+a u_{0}^{\eta}\right)\left(\frac{b}{r} u^{\xi}+a u^{\eta}\right)\right] f v_{1} \mathrm{~d} V \\
& +\oint_{\Gamma}\left(f_{s w}\left(u^{r}, w_{0}^{r} \boldsymbol{e}_{r}, \boldsymbol{n}\right)+\frac{1}{B} f_{s w}\left(u^{r}, w_{0}^{\xi} \boldsymbol{e}_{\xi}, \boldsymbol{n}\right)\right)\left[\left[v_{1}\right]\right] f \mathrm{~d} S \\
\mathcal{N}_{2}\left(\boldsymbol{u}, \boldsymbol{w}, v_{2}\right)= & \int_{\Omega}\left[u_{0}^{r} \partial_{r, h} u^{\eta}+\frac{1}{B} u_{0}^{\xi} \partial_{\xi, h} u^{\eta}+\frac{a^{2} B^{2}}{r} u_{0}^{r} u^{\eta}\right] f v_{2} \mathrm{~d} V \\
& +\oint_{\Gamma}\left(f_{s w}\left(u^{\eta}, w_{0}^{r} \boldsymbol{e}_{r}, \boldsymbol{n}\right)+\frac{1}{B} f_{s w}\left(u^{\eta}, w_{0}^{\xi} \boldsymbol{e}_{\xi}, \boldsymbol{n}\right)\right)\left[\left[v_{2}\right]\right] f \mathrm{~d} S \\
\mathcal{N}_{3}\left(\boldsymbol{u}, \boldsymbol{w}, v_{3}\right)= & \int_{\Omega}\left[u_{0}^{r} \partial_{r, h} u^{\xi}+\frac{1}{B} u_{0}^{\xi} \partial_{\xi, h} u^{\xi}+2 \frac{a b B^{2}}{r^{2}} u_{0}^{r} u^{\eta}+\frac{b^{2} B^{2}}{r^{3}} u_{0}^{r} u^{\xi}\right] f v_{3} \mathrm{~d} V \\
& +\oint_{\Gamma}\left(f_{s w}\left(u^{\xi}, w_{0}^{r} \boldsymbol{e}_{r}, \boldsymbol{n}\right)+\frac{1}{B} f_{s w}\left(u^{\xi}, w_{0}^{\xi} \boldsymbol{e}_{\xi}, \boldsymbol{n}\right)\right)\left[\left[v_{3}\right]\right] f \mathrm{~d} S
\end{aligned}
$$

To discretize the viscous terms, we employ a non-standard symmetric interior penalty (SIP) method to receive

$$
\begin{aligned}
\mathcal{A}_{1}\left(p, u, v_{1}\right)= & -v \int_{\Omega}\left[\left(-p+\partial_{r, h} u^{r}\right)\left(\frac{\mathrm{d} f}{\mathrm{~d} r} v_{1}+f \partial_{r, h} v_{1}\right)+u^{r}\left(\frac{\frac{\mathrm{d} f}{\mathrm{~d} r} \cdot r-f}{r^{2}} v_{1}+\frac{f}{r} \partial_{r, h} v_{1}\right)\right. \\
& \left.+\left(\frac{1}{B^{2}} \partial_{\xi} u^{r}+C^{r, \eta} u^{\eta}+C^{r, \xi} u^{\xi}\right) f \partial_{\xi, h} v_{1}-\frac{f}{r} u^{r} v_{1}\right] \mathrm{d} V \\
& +v \oint_{\Gamma}\left[\left(-p+\frac{1}{r}\left\{u^{r}\right\}+\left\{\partial_{r, h} u^{r}\right\}\right) \boldsymbol{e}_{r} \cdot \boldsymbol{n}\right. \\
& \left.+\left(\frac{1}{B^{2}}\left\{\partial_{\xi, h} u^{r}\right\}+C^{r, \eta}\left\{u^{\eta}\right\}+C^{r, \xi}\left\{u^{\xi}\right\}\right) \boldsymbol{e}_{\xi} \cdot \boldsymbol{n}\right] \cdot f\left[\left[v_{1}\right]\right] \mathrm{d} S
\end{aligned}
$$




$$
\begin{aligned}
& +v \oint_{\Gamma}\left(\left\{\partial_{r, h} v_{1}\right\} \boldsymbol{e}_{r}+\frac{1}{B^{2}}\left\{\partial_{\xi, h} v_{1}\right\} \boldsymbol{e}_{\xi}\right) \cdot \boldsymbol{n} f\left[\left[u^{r}\right]\right] \mathrm{d} S \\
& +v \oint_{\Gamma} \eta_{\operatorname{SIP}}\left[\left[u^{r}\right]\right]\left[\left[v_{1}\right]\right] \mathrm{d} S \text {, } \\
& \mathcal{A}_{2}\left(\boldsymbol{u}, v_{2}\right)=\int_{\Omega}\left[\partial_{r, h} u^{\eta}\left(\frac{\mathrm{d} f}{\mathrm{~d} r} v_{2}+f \partial_{r, h} v_{2}\right)+u^{\eta}\left(\frac{\frac{\mathrm{d} f}{\mathrm{~d} r} r-f}{r^{2}} v_{2}+\frac{f}{r} \partial_{r, h} v_{2}\right)\right. \\
& +\partial_{\xi, h} u^{\eta} \partial_{\xi, h} v_{2} \frac{f}{B^{2}}+u^{r} C^{\eta} f \partial_{\xi, h} v_{2}-B u^{\xi}\left(\partial_{r, h} C^{\eta} f v_{2}+C^{\eta} \frac{\mathrm{d} f}{\mathrm{~d} r} v_{2}+C^{\eta} f \partial_{r, h} v_{2}\right) \\
& \left.-\frac{a^{2} B^{2}\left(a^{2} B^{2}-2\right)}{r^{2}} u^{\eta} f v_{2}\right] \mathrm{d} V \\
& +\oint_{\Gamma}\left[\left(\left\{\partial_{r, h} u^{\eta}\right\}+\frac{1}{r}\left\{u^{\eta}\right\}-B\left\{u^{\xi}\right\}\right) \boldsymbol{e}_{r} \cdot \boldsymbol{n}\right. \\
& \left.+\left(\frac{1}{B^{2}}\left\{\partial_{\xi, h} u^{\eta}\right\}+C^{\eta}\left\{u^{r}\right\}\right) \boldsymbol{e}_{\xi} \cdot \boldsymbol{n}\right] \cdot f\left[\left[v_{2}\right]\right] \mathrm{d} S \\
& +v \oint_{\Gamma}\left(\left\{\partial_{r, h} v_{2}\right\} \boldsymbol{e}_{r}+\frac{1}{B^{2}}\left\{\partial_{\xi, h} v_{2}\right\} \boldsymbol{e}_{\xi}\right) \cdot \boldsymbol{n} f\left[\left[u^{\eta}\right]\right] \mathrm{d} S \\
& +v \oint_{\Gamma} \eta_{\operatorname{SIP}}\left[\left[u^{\eta}\right]\right]\left[\left[v_{2}\right]\right] \mathrm{d} S \text {, } \\
& \mathcal{A}_{3}\left(p, u, v_{3}\right)=-\int_{\Omega}\left[\left(-\frac{1}{B} p+\frac{1}{B^{2}} \partial_{\xi, h} u^{\xi}+C^{\xi, r} u^{r}\right) f \partial_{\xi, h} v_{3}\right. \\
& +\partial_{r, h} u^{\xi}\left(\frac{\mathrm{d} f}{\mathrm{~d} r} v_{3}+f \partial_{r, h} v_{3}\right)+u^{\xi}\left(\frac{\frac{\mathrm{d} f}{\mathrm{~d} r} r-f}{r^{2}} v_{3}+\frac{f}{r} \partial_{r, h} v_{3}\right) \\
& \left.+\frac{a B}{r} u^{\eta} C^{\xi, \eta}\left(\partial_{r, h} f v_{3}+\frac{\mathrm{d} f}{\mathrm{~d} r} v_{3}+f \partial_{r, h} v_{3}\right)-\frac{a^{4} B^{4}-1}{r^{2}} u^{\xi} f v_{3}\right] \mathrm{d} V \\
& +\oint_{\Gamma}\left[\left(\left\{\partial_{r, h} u^{\xi}\right\}+\frac{1}{r}\left\{u^{\xi}\right\}+C^{\xi, \eta} \frac{a B}{r}\left\{u^{\eta}\right\}\right) \boldsymbol{e}_{r} \cdot \boldsymbol{n}\right. \\
& \left.+\left(\frac{1}{B^{2}}\left\{\partial_{\xi, h} u^{\xi}\right\}+C^{\xi, r}\left\{u^{r}\right\}-\frac{1}{B} p\right) \boldsymbol{e}_{\xi} \cdot \boldsymbol{n} \cdot f\left[\left[v_{3}\right]\right]\right] \mathrm{d} S \\
& +v \oint_{\Gamma}\left(\left\{\partial_{r, h} v_{3}\right\} \boldsymbol{e}_{r}+\frac{1}{B^{2}}\left\{\partial_{\xi, h} v_{3}\right\} \boldsymbol{e}_{\xi}\right) \cdot \boldsymbol{n} f\left[\left[u^{\xi}\right]\right] \mathrm{d} S \\
& +v \oint_{\Gamma} \eta_{\operatorname{SIP}}\left[\left[u^{\xi}\right]\right]\left[\left[v_{3}\right]\right] \mathrm{d} S,
\end{aligned}
$$

where, for brevity, we use the following abbreviations for the coefficients

$$
\begin{array}{ll}
C^{r, \eta}:=-\frac{2 a b B}{r^{2}}, & C^{\xi, r}:=\frac{2 b^{2} B}{r^{3}}=-C^{r, \xi}, \quad C^{\eta}:=\frac{2 a b B}{r^{2}}=-C^{r, \eta}, \\
C^{r, \xi}:=-\frac{2 b^{2} B}{r^{3}}, \quad C^{\xi, \eta}:=\frac{2 b B}{r} .
\end{array}
$$


The first upper index corresponds to the momentum equation, whereas the second index indicates the corresponding velocity component. For example, $C^{r, \eta}$ is the coefficient of the $u^{\eta}$ term in the $r$-momentum equation.

\subsection{The temporal discretization}

For the temporal discretization we use a semi-explicit scheme, given in [37]. The main idea is to split the full spatial operator into a Stokes operator, which is discretized implicitly and the nonlinear term, which we implement explicitly. The reason for such a treatment of the temporal discretization is the time-independent uniform grid. Using that, we have a great advantage in performance: we need to solve the Stokes system only once and can compute many time steps with low computational costs by evaluating the convective terms explicitly. We use first and third order BDF scheme for the transient term and a third-order extrapolation (EX3) for the nonlinear term in case of the third order BDF scheme. For the uniform timestep size $\Delta t$ the Navier-Stokes equations (2.4) are discretized as follows

$$
\begin{aligned}
& \mathcal{C}\left(\boldsymbol{u}^{n+1}, \tau\right)=0 \quad \forall \tau \in \mathbb{V}_{k, 4^{\prime}}^{0} \\
& \frac{\beta_{0}}{\Delta t} \int_{\Omega} \boldsymbol{u}^{n+1} v \mathrm{~d} V+\mathcal{A}\left(p^{n+1}, \boldsymbol{u}^{n+1}, \boldsymbol{v}\right) \\
& \quad=\int_{\Omega}\left(\frac{\beta_{1}}{\Delta t} \boldsymbol{u}^{n}+\frac{\beta_{2}}{\Delta t} \boldsymbol{u}^{n-1}+\frac{\beta_{3}}{\Delta t} \boldsymbol{u}^{n-2}\right) \boldsymbol{v} \mathrm{d} V-\left(\gamma_{1} n\left(\boldsymbol{u}^{n}, \boldsymbol{w}^{n}, \boldsymbol{v}\right)+\gamma_{2} n\left(\boldsymbol{u}^{n-1}, \boldsymbol{w}^{n-1}, \boldsymbol{v}\right)\right. \\
& \left.\quad+\gamma_{3} n\left(\boldsymbol{u}^{n-2}, \boldsymbol{w}^{n-2}, \boldsymbol{v}\right)\right) \quad \forall \boldsymbol{v} \in \mathbb{V}_{k, i^{\prime}}^{0}, i=1, \cdots 3
\end{aligned}
$$

where $\mathcal{A}$ is the bilinearform (3.20), $\mathbb{V}_{k, i}^{0}$ is the DG space defined in (3.13) and $\mathcal{C}$ is the discretized velocity divergence, given by (3.15). For a BDF scheme of first order the coefficients are given by $\beta_{0}=1, \beta_{1}=\beta_{2}=\beta_{3}=0$ and $\gamma_{1}=1, \gamma_{2}=\gamma_{3}=0$, whereas for a BDF scheme of third order the coefficients are given by $\beta_{0}=\frac{11}{6}, \beta_{1}=3, \beta_{2}=-\frac{3}{2}, \beta_{3}=\frac{1}{3}$ and $\gamma_{1}=3, \gamma_{2}=-3, \gamma_{3}=1$ for the third-order extrapolation (EX3). The key advantage of this method is that the left-hand-side of $(3.22 b)$ is constant in time. Thus, we can use a direct solver and save the factorization which means that a computationally expensive factorization must be done only once.

\section{Convergence studies on a cylindrical shell and results}

We first discretize the helically invariant Navier-Stokes equations on a cylindrical shell, which obviously avoids singularities at $r=0$. A cylindrical shell means that we restrict the radial coordinate $r$ to be non-zero and hence the computational parameter domain is given by $\Omega=[r \times \xi]=[0.1 \cdots 1 \times 0 \cdots 2 \pi]$. In this case, we restrict the metric function to $f(r)=1$ such that a natural DG discretization may be implemented. The discretization on a full cylindrical domain including the centerline axis will be presented in Section 5. 


\subsection{Test setup}

As a test case for the numerical implementation of the helically invariant system of Navier-Stokes equations we consider the following time-dependent manufactured solution, which is given by

$$
\begin{aligned}
& u^{r}=\left(1-e^{-r^{2}}\right) \sin (\xi) \cos (t), \\
& u^{\xi}=\left(2 r B e^{-r^{2}} \cos \xi+\frac{B}{r}\left(1-e^{-r^{2}}\right) \cos (\xi)\right) \cos (t), \\
& u^{\eta}=\left(1-e^{-r^{2}}\right) \cos (\xi) \cos (t), \\
& p=\left(1-e^{-r^{2}}\right) \sin (\xi) \cos (t) .
\end{aligned}
$$

The present solution is periodic in $\xi$-direction and thus fulfills the periodicity condition

$$
u^{j}(r, \xi)=u^{j}(r, \xi+2 \pi)
$$

and

$$
p(r, \xi)=p(r, \xi+2 \pi) .
$$

Note that the manufactured solution (4.1) is divergence-free, i.e. the continuity equation is fulfilled. For this ansatz residual terms arise, which are implemented as source terms in the helically invariant Navier-Stokes system. For testing the steady Navier-Stokes system, we reduce the manufactured solution (4.1) by assuming $\cos (t)=1$, which leads to the following expressions for the velocity and pressure

$$
\begin{aligned}
& u^{r}=\left(1-e^{-r^{2}}\right) \sin (\xi), \\
& u^{\xi}=2 r B e^{-r^{2}} \cos \xi+\frac{B}{r}\left(1-e^{-r^{2}}\right) \cos (\xi), \\
& u^{\eta}=\left(1-e^{-r^{2}}\right) \cos (\xi), \\
& p=\left(1-e^{-r^{2}}\right) \sin (\xi) .
\end{aligned}
$$

\subsection{Grid refinement study}

In this section we first consider the spatial convergence of the steady helically invariant Navier-Stokes equations, using the manufactured solution (4.4) at $t=0$. Furthermore, we perform a temporal convergence study for the transient case, for which we use the time-dependent manufactured solution (4.1). 


\subsubsection{Spatial convergence study}

For the spatial convergence study we consider a uniform Cartesian grid with $4 \times 4$ cells for the coarsest and $64 \times 64$ cells for the finest one. Since the computational domain is rectangular, each cell also has a rectangular shape and we choose the shortest edge as the cell size, i.e. $h=\min (\mathrm{d} r, \mathrm{~d} \xi)$. The convergence study has been driven for each component of the helical velocity vector $\mathbf{u}$ as well as for the pressure $p$. We investigate different polynomial orders, varying form $k=1$ to $k=3$ for the velocity and $k^{\prime}=0, \cdots, 2$ for the pressure. For each DG degree and each velocity component we obtain the convergence rate of $k+1$, which we expect from theory. For the pressure $p$ we observe super-convergence due to a highly regular mesh on the whole fluid domain and the fact that we use the exact solution (4.4) of the problem on all boundaries (cf. Fig. 2).

In addition to that, we present an equal order formulation of the spatial discretization of the system (2.9). In order to control pressure jumps across interfaces, we add a stability term to the continuity equation (3.14a), as proposed in [13]. Hence, the continuity equation reads

$$
c(u, \tau)+\lambda(p, \tau)=0 \quad \forall \tau \in \mathbb{V}_{k}^{0}
$$


Figure 2: $L^{2}$ norm errors, measured with respect to the manufactured solution (4.4) for the velocity $u$ and pressure $p$ v.s. the grid size $h$. For comparison the solid lines are plotted to show the slopes $m=(3,4,5)$ for the velocity and $m=(2,3,4)$ for the pressure, respectively. Computations for $4 \times 4, \cdots, 64 \times 64$ cells and DG degrees $k=1, \cdots, 3$ and $k^{\prime}=0, \cdots, 2$ on a domain $\Omega=[r \times \xi]=[0.1, \cdots, 1 \times 0, \cdots, 2 \pi]$. Mixed order formulation. 

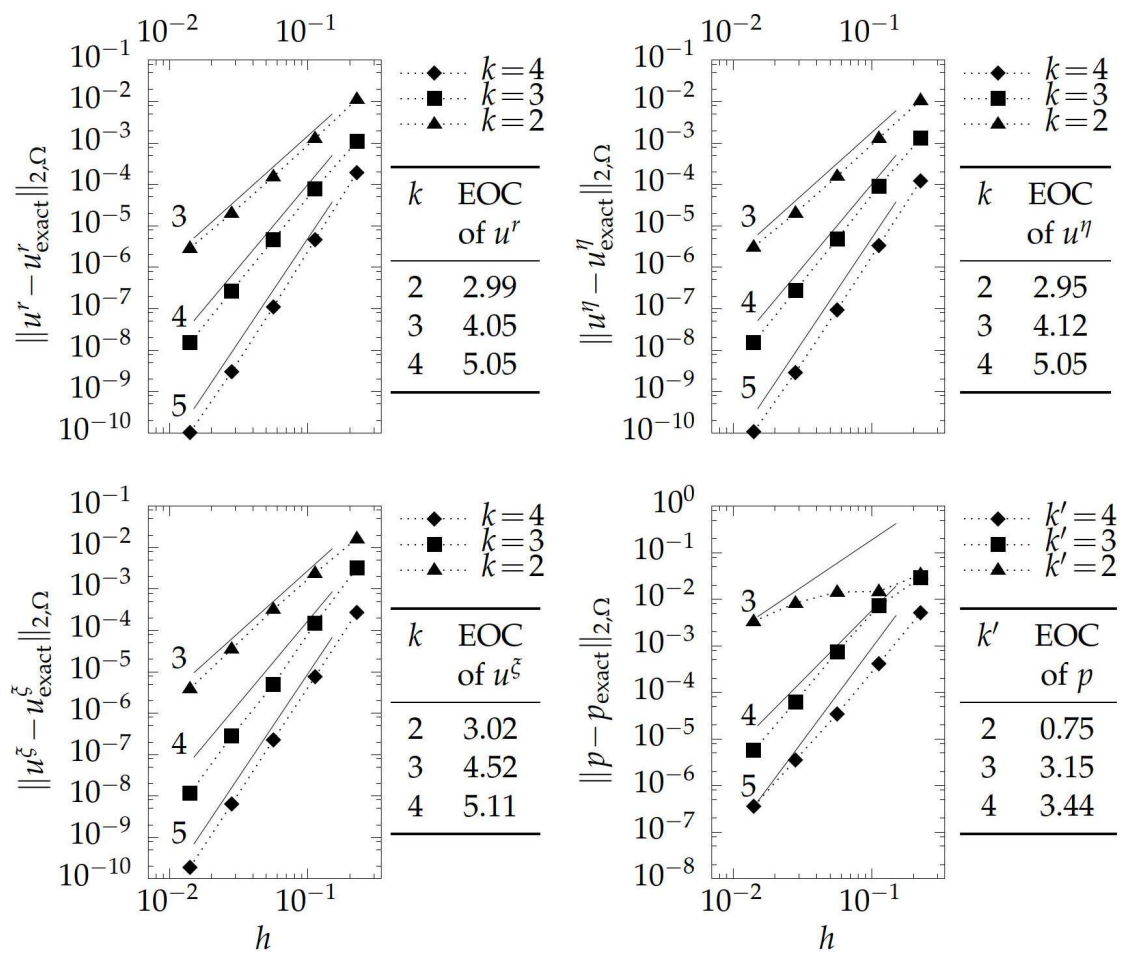

Figure 3: $L^{2}$ norm errors, measured with respect to the manufactured solution (4.4) for the velocity $u$ and pressure $p$ v.s. the grid size $h$. Computations for $4 \times 4, \cdots, 64 \times 64$ cells and DG degrees $k=k^{\prime}=2, \cdots, 4$ on a domain $\Omega=[r \times \xi]=[0.1, \cdots, 1 \times 0, \cdots, 2 \pi]$. Equal order formulation with pressure stabilization in the continuity equation (4.5).

where the stabilization term is given by

$$
\lambda(p, \tau):=\oint_{\Gamma} h_{\Gamma}[[p]][[\tau]] f(r) \mathrm{d} S .
$$

Herein, $h_{F}$ is a local length scale for which we choose the longest edge of each cell, i.e. $\max (\Delta r, \Delta \xi)$. As before, $f(r)$ is the metric function, which is set to $f(r)=1$ for computations on the cylindrical shell. Fig. 3 shows the results for a $h$-convergence study, analogously to the studies for the mixed-order DG formulation in Fig. 2. Comparing both convergence studies, the results for the velocity components are rather similar. Concerning the pressure the mixed-order formulation shows better results for low DG degrees, whereas for DG degree $k=3$ also the equal order formulation reaches approximately the expected convergence rate. Due to these results, we subsequently will keep the focus on the mixed-order formulation.

\subsubsection{Temporal convergence study}

For the temporal discretization we consider a BDF scheme of third order with time step sizes from $\Delta t=3.125 \times 10^{-2}$ to $\Delta t=0.25$. The polynomial order is chosen to $k=4$ for 



Figure 4: Temporal convergence studies, using the test case (4.1). The $L^{2}$ norm errors are plotted for the velocity $u$ and pressure $p$ v.s. the time step size $\Delta t$. Computations for $32 \times 32$ cells and DG degrees $k=4$ and $k^{\prime}=3$ on a domain $\Omega=[r \times \xi]=[0.1, \cdots, 1 \times 0, \cdots, 2 \pi]$. Mixed order formulation.

the velocity and $k^{\prime}=3$ for the pressure and all computations are conducted on a grid of $32 \times 32$ cells, such that the temporal error dominates the error of the spatial operator. For all physical quantities, i.e. all velocity components and the pressure the expected convergence rate of three is approximately obtained (cf. Fig. 4).

The $L^{2}$ norm errors for the velocity $e_{u}$ and the pressure $e_{p}$ are calculated as follows

$$
e_{u}=\left\|\boldsymbol{u}^{n_{s}}-\boldsymbol{u}_{h}\left(n_{s} \Delta t\right)\right\|_{L^{2}(\Omega)}^{2}, \quad e_{p}=\left\|p^{n_{s}}-p_{h}\left(n_{s} \Delta t\right)\right\|_{L^{2}(\Omega)}^{2},
$$

where $\boldsymbol{u}^{n_{s}}, p^{n_{s}}$ is the exact solution at $t=n_{s} \Delta t$.

\section{Convergence studies on the full cylindrical domain and results}

In this section we consider the DG discretization of the helically invariant Navier-Stokes equations (2.9) on the full cylindrical domain, including the centerline axis at $r=0$. In this part of the fluid domain two additional conditions have to be considered. The first condition is obtained from the periodicity in $\xi$-direction of the helical flow. Considering the invariant coordinate (2.2), for both helical coordinates $\xi$ and $\eta$ periodicity condi- 
tions $\xi_{p}=2 \pi b, \xi_{l}=\frac{2 \pi r^{2} a^{2}}{b}$ and $\eta_{p}=-2 \pi r^{2} a$ can be derived which is shown in Appendix $\mathrm{B}$. The geometrical length of the helix $p$ depends on the radial coordinate and is given by $p=2 \pi b B$ which becomes zero at the centerline, since (2.8) holds. A zero length of the helix would mean that there are infinitely many helical turns at the centerline axis. Hence, we conclude that all velocity components and the pressure must be $\xi$-independent, i.e. $\frac{\partial}{\partial \xi}=0$ at $r=0$. The second condition that we need is one for the uniqueness of all dependent variables at $r=0$. For that, we follow [29] who considered the following conditions at the centerline in cylindrical coordinates, given by

$$
\lim _{r \rightarrow 0} \frac{\partial u}{\partial \varphi}=0, \quad \lim _{r \rightarrow 0} \frac{\partial p^{\prime}}{\partial \varphi}=0 .
$$

For $r>0$, one may consider a ring around the $z$-axis where different velocities in circumferential direction arise at each point. For $r \rightarrow 0$ this circle reduces to a point at the $z$-axis, where a unique velocity must be given. This leads to the condition that the velocity must not change in circumferential direction for $r \rightarrow 0$, which implies (5.1). A transformation of the conditions (5.1) into the helical frame leads to the following conditions for the helical variables

$$
b u_{\xi}^{r}=u^{\xi}, \quad b u_{\xi}^{\xi}=-u^{r}, \quad u_{\xi}^{\eta}=0, \quad p_{\xi}=0 .
$$

The derivation of the conditions (5.2) is presented in Appendix C. Combining the periodicity and uniqueness conditions finally leads to the following centerline conditions, given by

$$
\text { (a) } u^{r}=0, \quad \text { (b) } u^{\xi}=0, \quad \text { (c) } u_{\xi}^{\eta}=0, \quad \text { (d) } p_{\xi}=0,
$$

which means that the radial and helical velocity components vanish whereas the velocity component in invariant direction and the pressure are constant with respect to spatial coordinates. In the following section we present a method to reduce the DOFs of the DG discretization at $r=0$, which is equivalent to a change of the DG basis and arises from the conditions (5.3).

\subsection{Implementation of the reduced DG space $\mathbb{V}_{K}^{0}$}

In each timestep the evaluation of the spatial operator and the matrix assembly rely on the DG space $\mathbb{V}_{k}$. Since the reduced DG space $\mathbb{V}_{k}^{0}$ is a linear subspace of the DG space $\mathbb{V}_{k}$, i.e. $\mathbb{V}_{k}^{0} \leq_{\mathbb{R}} \mathbb{V}_{k}$, a basis $\Psi^{B}$ of $\mathbb{V}_{k}^{0}$ can readily be written as $\Psi^{B}=\boldsymbol{\Phi}^{B} \cdot \boldsymbol{A}$, where $\boldsymbol{\Phi}^{B}$ is a basis of $\mathbb{V}_{k}$ and $A \in \mathbb{R}^{\operatorname{dim}\left(\mathbb{V}_{k}\right) \times \operatorname{dim}\left(\mathbb{V}_{k}^{0}\right)}$ is a non-quadratic matrix. The reduction of the DG space can be done component-wise for each component of the velocity vector $u$ as well as for the pressure $p$. In the following we distinguish two different cases of DG space reductions. In the first case we discuss the DG space reduction using the conditions $(5.3 \mathrm{a}, \mathrm{b})$, which are cell-local conditions. The second case is a global DG space reduction where the conditions, given by $(5.3 \mathrm{c}, \mathrm{d})$ are used. Hence the first case is more restrictive and is implemented locally on each cell at the centerline, whereas the second case represents a global relation connecting the neighboring cells in $\xi$-direction at $r=0$. 


\subsection{Case 1: Cell-local reduction of the DG space $\mathbb{V}_{k}$}

In this first case the two centerline conditions $u^{r}=0$ and $u^{\xi}=0$ are implemented. Let $\left(\Phi_{j, 1}^{B}, \cdots, \Phi_{j, N_{k}}\right)$ be a basis of a DG space of degree $k$ in cell $K_{j}$ which is located at the centerline $r=0$, i.e. $\overline{K_{j}} \cap(\{0\} \times \mathbb{R}) \neq 0$. For both velocity components $u^{i}$, with $i=\{r, \xi\}$, we consider the ansatz $u^{i}(r, \xi)=\sum_{n=1}^{N_{k}} \Phi_{j, n}^{B}(r, \xi) \tilde{u}_{j, n}^{i} \stackrel{!}{=} 0$ for $r=0$ and the nodes at the centerline in $K_{j}$ which are $\xi=\xi_{1}, \xi_{2}, \cdots, \xi_{N_{k}+1}$, where $\xi_{1}<\xi_{2}<\cdots<\xi_{N_{k}+1}$ and $\left(0, \xi_{k}\right) \in \overline{K_{j}}$. The ansatz leads to a system of equations, given by

$$
\underbrace{\left[\begin{array}{ccc}
\Phi_{j, 1}^{B}\left(0, \xi_{1}\right) & \cdots & \Phi_{j, N_{k}}^{B}\left(0, \xi_{1}\right) \\
\vdots & & \vdots \\
\Phi_{j, 1}^{B}\left(0, \xi_{N_{k}+1}\right) & \cdots & \Phi_{j, N_{k}}^{B}\left(0, \xi_{N_{k}+1}\right)
\end{array}\right]}_{B}\left[\begin{array}{c}
\tilde{u}_{j, 1}^{i} \\
\vdots \\
\tilde{u}_{j, N_{k}}^{i}
\end{array}\right]=\mathbf{0},
$$

for the velocity components $u^{r}$ and $u^{\xi}$. Eq. (5.4) is a linear system of the form $\boldsymbol{B} \tilde{\boldsymbol{u}}=\mathbf{0}$, where $B$ denotes the matrix of the basis functions, evaluated at the centerline $r=0$. In a next step we need to find a matrix $A \in \mathbb{R}^{\operatorname{dim}\left(\mathbb{V}_{k}\right) \times \operatorname{dim}\left(\mathbb{V}_{k}^{0}\right)}$ such that the columns of $A$ represent a solution of (5.4), i.e. $\boldsymbol{B} \cdot \boldsymbol{A}=\mathbf{0}$. Using that, it follows that

$$
\left(\Psi_{j, 1}, \cdots, \Psi_{j, l}\right)=\left(\Phi_{j, 1}, \cdots, \Phi_{j, N_{k}}\right) A
$$

is a basis which fulfills the centerline conditions (5.3a,b). The matrix $A$ can be obtained e.g. from the row echelon form of $\boldsymbol{B}$, which is a generalized Gaussian elimination.

\subsection{Case 2: Global reduction of the DG space $\mathbb{V}_{k}$}

In the second case the centerline conditions, given by $(5.3 c, d)$ are implemented. The implementation is divided into two steps. In the first step we proceed in analogy to the first case. The DG space is locally reduced in each cell at the centerline but this time using the $\xi$-derivative of the basis functions $\partial_{\tilde{\zeta}} \Phi_{j, k}^{B}$. Hence, in this case the system of equations $B \tilde{u}=\mathbf{0}$ is given by

$$
\left[\begin{array}{ccc}
\partial_{\xi} \Phi_{j, 1}^{B}\left(0, \xi_{1}\right) & \cdots & \partial_{\tilde{\xi}} \Phi_{j, N_{k}}^{B}\left(0, \xi_{1}\right) \\
\vdots & & \vdots \\
\partial_{\xi} \Phi_{j, 1}^{B}\left(0, \xi_{N_{k}+1}\right) & \cdots & \partial_{\xi} \Phi_{j, N_{k}}^{B}\left(0, \xi_{N_{k}+1}\right)
\end{array}\right]\left[\begin{array}{c}
\tilde{u}_{j, 1}^{i} \\
\vdots \\
\tilde{u}_{j, N_{k}}^{i}
\end{array}\right]=\mathbf{0 .}
$$

From that, a matrix $A^{*}$ can be obtained in analogy to the proceeding in Section 5.2. In the second step we connect the neighboring cells at $r=0$. For that, we consider two cells $K_{j}$ and $K_{l}$ and demand that

$$
u^{\eta}\left(0, \xi_{1}\right)=u^{\eta}\left(0, \xi_{2}\right)
$$


for nodes $\left(0, \xi_{1}\right) \in \overline{K_{j}}$ and $\left(0, \xi_{2}\right) \in \overline{K_{l}}$. The same condition is used for the pressure $p$. Using the polynomial DG ansatz, (5.7) can be written as

$$
[\underbrace{\Phi_{j, 1}^{B}\left(0, \xi_{1}\right), \cdots, \Phi_{j, N_{k}}^{B}\left(0, \xi_{1}\right),-\Phi_{l, 1}^{B}\left(0, \xi_{2}\right), \cdots,-\Phi_{l, N_{k}}^{B}\left(0, \xi_{2}\right)}_{\left[B_{1}^{*},-B_{2}^{*}\right]}] \cdot\left[\begin{array}{c}
\tilde{u}_{j, 1}^{\eta} \\
\vdots \\
\tilde{u}_{j, N_{k}}^{\eta} \\
\tilde{u}_{l, 1}^{\eta} \\
\vdots \\
\tilde{u}_{l, N_{k}}^{\eta}
\end{array}\right]=\mathbf{0} .
$$

Just as before, a matrix $R$ needs to be determined such that the following transformation holds

$$
\underbrace{\left[\begin{array}{cc}
\boldsymbol{I} & \mathbf{0} \\
\boldsymbol{R}_{21} & \boldsymbol{R}_{22}
\end{array}\right]}_{\boldsymbol{R}}\left[\begin{array}{c}
\tilde{u}_{j, 1}^{\eta} \\
\vdots \\
\tilde{u}_{j, N_{k}}^{\eta} \\
\tilde{u}_{l, 1}^{\eta} \\
\vdots \\
\tilde{u}_{l, N_{k}}^{\eta}
\end{array}\right]=\left[\begin{array}{c}
\tilde{u}_{j, 1}^{* \eta} \\
\vdots \\
\tilde{u}_{j, N_{k}}^{* \eta} \\
\tilde{u}_{l, 1}^{* \eta} \\
\vdots \\
\tilde{u}_{l, N_{k}}^{* \eta}
\end{array}\right]
$$

where $\tilde{u}_{j, 1}^{* \eta}, \cdots, \tilde{u}_{l, N_{k}}^{* \eta}$ are the DG coordinates in the reduced DG space. The transformation is constructed as such that the coordinates in cell $K_{j}$ are maintained. Similar to Section 5.2 we find the solution space of $\left[\boldsymbol{B}_{1}^{*},-\boldsymbol{B}_{2}^{*}\right]$ through a solution of

$$
\left[B_{1}^{*},-B_{2}^{*}\right]\left[\begin{array}{cc}
I & 0 \\
R_{21} & R_{22}
\end{array}\right]=\mathbf{0}
$$

It follows that the block $\boldsymbol{R}_{22}$ is determined by the solution space of $\boldsymbol{B}_{2}^{*}$ and $\boldsymbol{R}_{21}$ can be obtained by the solution of

$$
B_{2}^{*} \cdot R_{21}=B_{1}^{*},
$$

using the least squares method for underdetermined systems. The final transformation matrix $Q$ that combines both steps (i) the cell-local reduction by evaluation of the $\xi$ derivatives of the basis functions and (ii) the reduction connecting neighbor cells is given by

$$
Q=A^{*} \cdot R
$$

\subsection{Grid refinement study}

As before for the cylindrical shell domain, we present a spatial and temporal convergence study for our test case (4.4). Since in the full cylindrical domain the helically invariant 
Navier-Stokes equations (2.9) are solved using a Petrov-Galerkin method, we introduce the following norm, given by

$$
\left\|u^{i}-u_{\text {exact }}^{i}\right\|_{B}:=\left\|B(r)\left(u^{i}-u_{\text {exact }}^{i}\right)\right\|_{2}
$$

where the numerical errors are scaled by the metric function $B(r)$ defined in (2.8) and the $u^{i}$ denote the components of the velocity vector, i.e. $u^{r}, u^{\tau}$ and $u^{\eta}$.

Since the pressure is only unique up to a constant $c$, we need to find $c$ such that

$$
\int_{\Omega}\left(p_{\text {err }}-c\right)^{2} B(r)^{2} \mathrm{~d} \Omega \rightarrow \min ,
$$

where $p_{\text {err }}=p-p_{\text {exact }}$. A quick calculation shows that

$$
c=\frac{\int_{\Omega} p_{e r r} B(r)^{2} \mathrm{~d} \Omega}{\int_{\Omega} B(r)^{2} \mathrm{~d} \Omega} .
$$

\subsubsection{Spatial convergence study}

In Fig. 5 convergence plots for the mixed order formulation are presented. In particular for DG degrees $k=3$ and $k=4$, the convergence rates are very close to what we expect
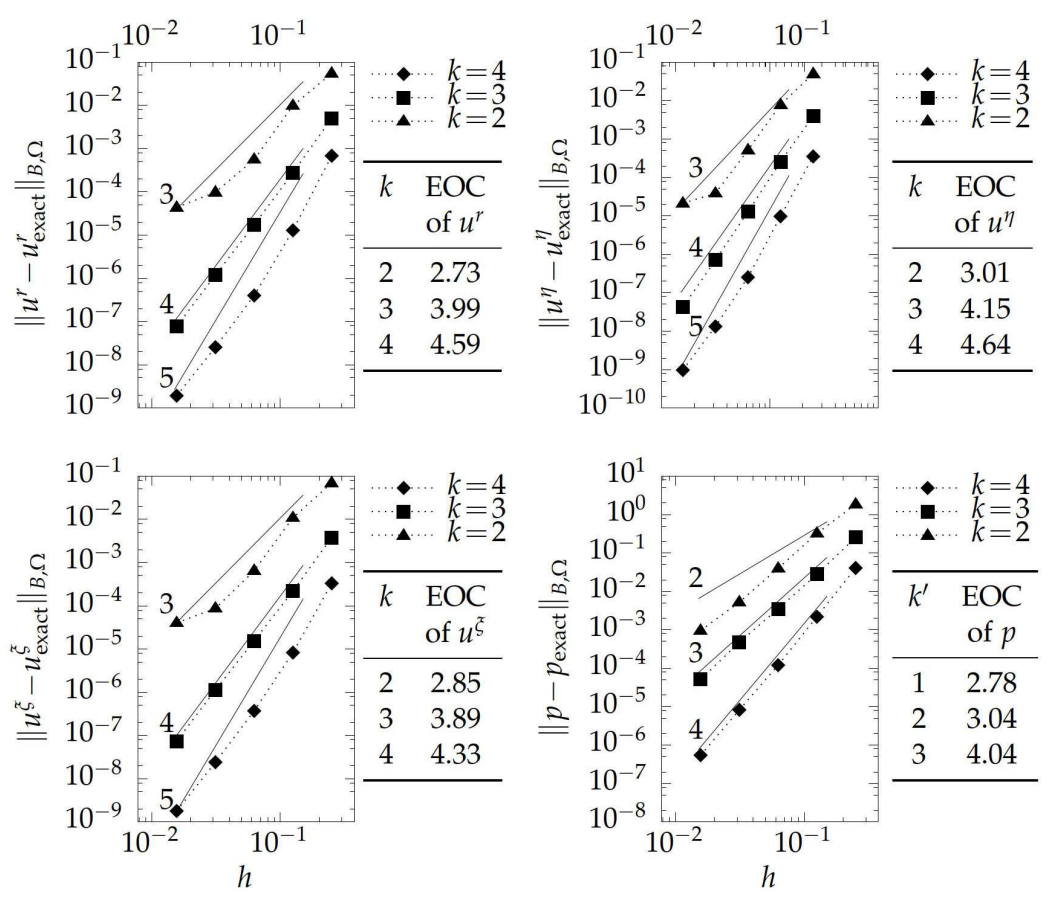

Figure 5: Numerical errors, measured in the $B$-norm defined by (5.13) with respect to the manufactured solution (4.4). The errors for the velocity $u$ and pressure $p$ are plotted v.s. the grid size $h$. Computations for $4 \times$ $4, \cdots, 64 \times 64$ cells and DG degrees $k=2 \cdots 4$ and $k^{\prime}=1, \cdots, 3$ on the full domain $\Omega=[r \times \xi]=[0, \cdots, 1 \times 0, \cdots, 2 \pi]$. Mixed order formulation. 

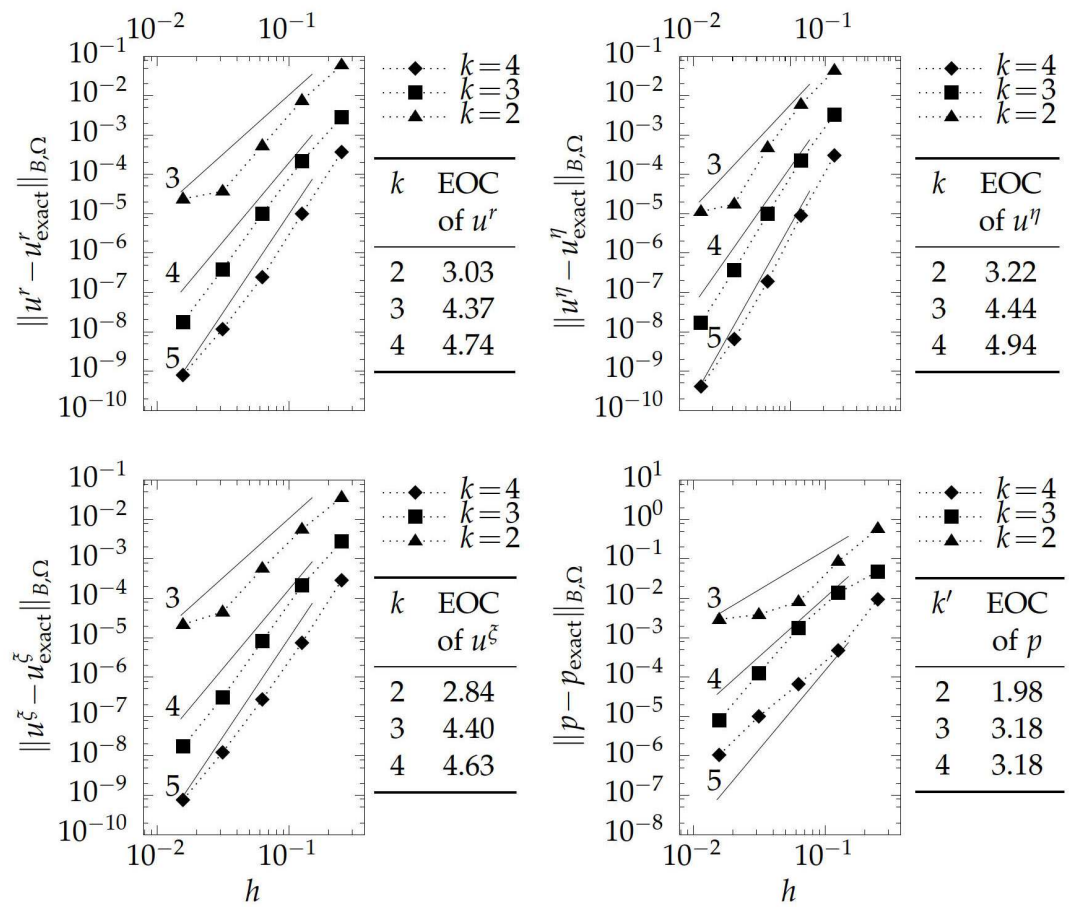

Figure 6: Numerical errors, measured in the B-norm defined by (5.13) with respect to the manufactured solution (4.4). The errors for the velocity $u$ and pressure $p$ are plotted v.s. the grid size $h$. Computations for $4 \times$ $4, \cdots, 64 \times 64$ cells and DG degrees $k=k^{\prime}=2, \cdots, 4$ on the full domain $\Omega=[r \times \xi]=[0, \cdots, 1 \times 0, \cdots, 2 \pi]$. Equal order formulation.

from the theory. Fig. 6 shows convergence plots for the equal order formulation. Here, also for DG degrees $k=3$ and $k=4$ reach approximately the expected convergence rate.

\subsubsection{Temporal convergence study}

As before in Section 4.2.2, we consider the temporal convergence rates of the transient numerical discretization using the semi-explicit BDF3 scheme introduced in Section 3.3. For time step sizes from $\Delta t=3 \times 10^{-2}$ to $\Delta t=0.25$, the polynomial order $k=4$ and $k^{\prime}=3$ for the velocity and pressure and a spatial grid consisting of $32 \times 32$ cells, we obtain the expected convergence rate for a third-order time integration scheme on the full cylindrical domain, which is shown in Fig. 7.

\section{Conclusions and outlook}

In this article, a numerical code to solve the helically invariant Navier-Stokes equations has been developed. The numerical discretization is based on the high-order discontinuous Galerkin method on a structured quadrilateral mesh. The computational domain is a reduced parameter set consisting of the radial coordinate $r$ and the helical coordi- 

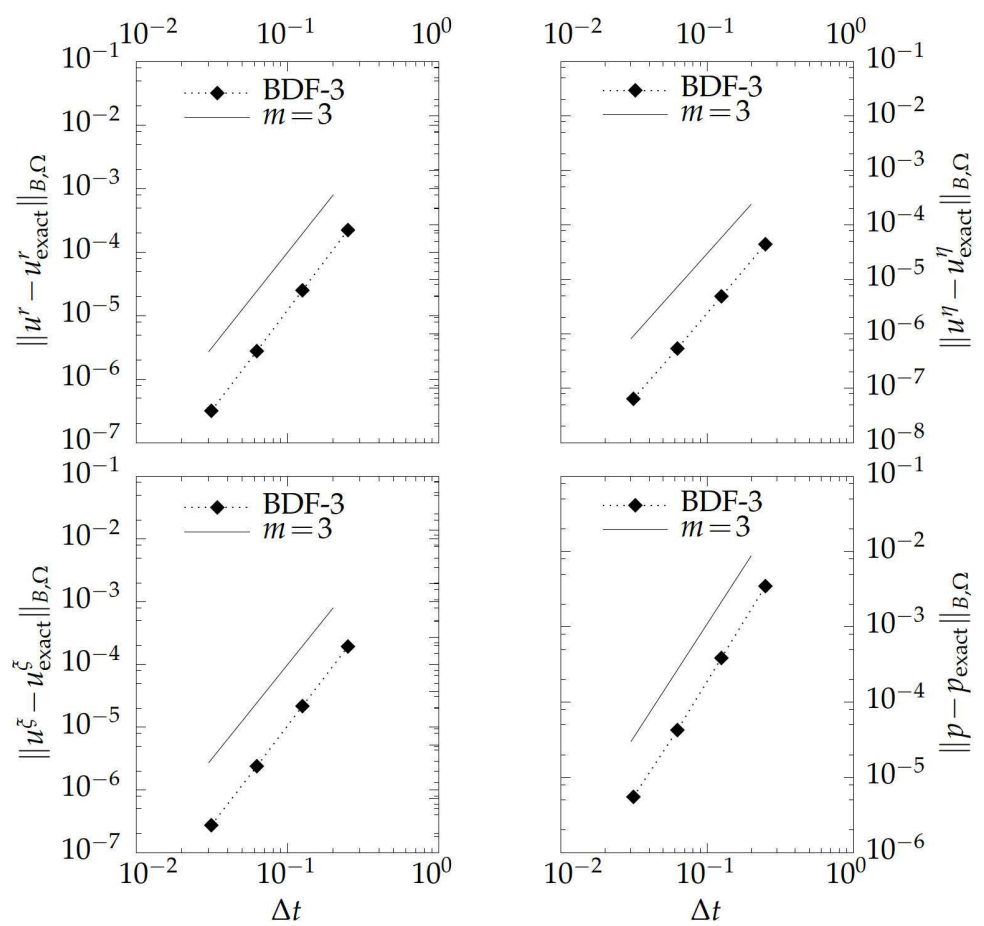

Figure 7: Temporal convergence studies, using the test setup (4.1). The numerical errors are measured in the $B$-norm, defined by (5.13). The velocity $u$ and pressure $p$ is plotted v.s. the time step size $\Delta t$. Computations for $32 \times 32$ cells and DG degrees $k=4$ and $k^{\prime}=3$ on a full cylindrical domain $\Omega=[r \times \xi]=[0, \cdots, 1 \times 0, \cdots, 2 \pi]$. Mixed order formulation.

nate $\xi$ which describes a helical line. Considering a grid refinement study, we show that the present spatial discretization using a high order polynomial approximation up to order $k=4$ reaches an accuracy of $\mathcal{O}\left(h^{k+1}\right)$, which is expected from DG theory. For the time integration, a semi-explicit time-stepper has been implemented where a third order BDF scheme for the transient term and a third-order extrapolation (EX3) for the nonlinear term has been used. Temporal convergence studies show the expected convergence rates. The numerical tests have been conducted on a cylindrical shell as well as on the full cylindrical domain where the centerline axis at $r=0$ is included. On the full domain additional conditions for uniqueness are implemented resulting in a reduction of degrees of freedom at $r=0$ and hence a change of the DG basis at the origin.

The central application of the newly developed code is vortex dynamics and turbulence research where numerical simulations of dimensional reduced flows are of highest interest. It is well-known that in two-dimensional turbulence physical mechanisms are significantly different to those in three dimensions. For example, in three dimensional turbulence energy is transferred from the large to the small eddies and a stretching of the vortices appears, whereas in two dimensions the vortex stretching vanishes and the energy is transferred in opposite direction. As introduced at the beginning of this arti- 
cle, helically reduced flows are placed between two and three dimensions and to date it is not known in which direction energy is transferred. Using the present numerical discretization, simulations with high Reynolds numbers will lead to energy spectra that give answers to the question of energy transport of helically symmetric flows and to many other questions concerning the behavior of two and three dimensional turbulence.

\section{Acknowledgments}

The authors are grateful to the Germany Research Foundation (DFG) under grant no. OB 96/41-1 for financial support and to M. Oberlack for his helpful comments and various inspiring discussions.

\section{A Derivation of a new orthogonal helically invariant coordinate}

Due to the singular behaviour of the helical coordinate $\tilde{\eta}$ at the origin $r=0$, originally introduced by $\mathrm{KCO}$, a new third coordinate is derived in the following. This coordinate is needed to formulate well-defined periodicity conditions at the centerline axis $r=0$, that are necessary for the implementation of periodic boundary conditions in the DG discretization of the helically invariant Navier-Stokes equations.

We use the two helical coordinates introduced in [28]

$$
\tilde{r}=r, \quad \xi=a z+b \varphi
$$

and attend to find a third coordinate $\eta$ in the invariant direction. From (A.1) one may determine the Jacobian matrix

$$
J=\left[\begin{array}{lll}
\frac{\partial \tilde{r}}{\partial r} & \frac{\partial \tilde{r}}{\partial \varphi} & \frac{\partial \tilde{r}}{\partial z} \\
\frac{\partial \tilde{\zeta}}{\partial r} & \frac{\partial \mathcal{\zeta}}{\partial \varphi} & \frac{\partial \tilde{\zeta}}{\partial z} \\
\frac{\partial \eta}{\partial r} & \frac{\partial \eta}{\partial \varphi} & \frac{\partial \eta}{\partial z}
\end{array}\right]=\left[\begin{array}{ccc}
1 & 0 & 0 \\
0 & a & b \\
\frac{\partial \eta}{\partial r} & \frac{\partial \eta}{\partial \varphi} & \frac{\partial \eta}{\partial z}
\end{array}\right]
$$

The inverse relation reads

$$
J^{-1}=\left[\begin{array}{lll}
\frac{\partial r}{\partial \tilde{r}} & \frac{\partial r}{\partial \tilde{\zeta}} & \frac{\partial r}{\partial \eta} \\
\frac{\partial \varphi}{\partial \tilde{r}} & \frac{\partial \varphi}{\partial \zeta} & \frac{\partial \varphi}{\partial \eta} \\
\frac{\partial z}{\partial \tilde{r}} & \frac{\partial z}{\partial \tilde{\zeta}} & \frac{\partial z}{\partial \eta}
\end{array}\right],
$$

which can be obtained by inverting (A.2). We have

$$
J^{-1}=\frac{1}{b \frac{\partial \eta}{\partial z}-a \frac{\partial \eta}{\partial \varphi}}\left[\begin{array}{ccc}
1 & 0 & 0 \\
a \frac{\partial \eta}{\partial r} & \frac{\partial \eta}{\partial z} & -a \\
-b \frac{\partial \eta}{\partial r} & \frac{\partial \eta}{\partial \varphi} & b
\end{array}\right]
$$


Comparing (A.3) and (A.4) leads to

$$
\begin{array}{ll}
\frac{\partial \varphi}{\partial \xi}=\frac{\frac{\partial \eta}{\partial z}}{b \frac{\partial \eta}{\partial z}-a \frac{\partial \eta}{\partial \varphi}}, & \frac{\partial \varphi}{\partial \eta}=\frac{-a}{b \frac{\partial \eta}{\partial z}-a \frac{\partial \eta}{\partial \varphi}}, \\
\frac{\partial z}{\partial \xi}=\frac{-\frac{\partial \eta}{\partial \varphi}}{b \frac{\partial \eta}{\partial z}-a \frac{\partial \eta}{\partial \varphi}}, & \frac{\partial z}{\partial \eta}=\frac{b}{b \frac{\partial \eta}{\partial z}-a \frac{\partial \eta}{\partial \varphi}} .
\end{array}
$$

The derivatives are given by

$$
\begin{aligned}
& \partial_{\eta}=\frac{\partial r}{\partial \eta} \partial_{r}+\frac{\partial \varphi}{\partial \eta} \partial_{\varphi}+\frac{\partial z}{\partial \eta} \partial_{z} \\
& \partial_{\xi}=\frac{\partial r}{\partial \xi} \partial_{r}+\frac{\partial \varphi}{\partial \xi} \partial_{\varphi}+\frac{\partial z}{\partial \xi} \partial_{z} .
\end{aligned}
$$

For the helical and cylindrical coordinates we know that

$$
\frac{\partial r}{\partial \eta}=0, \quad \frac{\partial r}{\partial \xi}=0, \quad \partial_{\varphi}=r \boldsymbol{e}_{\varphi}, \quad \partial_{z}=\boldsymbol{e}_{z}
$$

For orthogonality of the coordinate lines $\xi=$ const. and $\eta=$ const. the condition $\partial_{\xi} \cdot \partial_{\eta}=0$ must be fulfilled. That leads to a determining PDE for the coordinate $\eta$, given by

$$
\begin{aligned}
\partial_{\xi} \cdot \partial_{\eta} & =\frac{\partial \varphi}{\partial \xi} \frac{\partial \varphi}{\partial \eta} r^{2}+\frac{\partial z}{\partial \xi} \frac{\partial z}{\partial \eta} \\
& =\frac{-a r^{2} \frac{\partial \eta}{\partial z}}{\left(b \frac{\partial \eta}{\partial z}-a \frac{\partial \eta}{\partial \varphi}\right)^{2}}+\frac{-b \frac{\partial \eta}{\partial \varphi}}{\left(b \frac{\partial \eta}{\partial z}-a \frac{\partial \eta}{\partial \varphi}\right)^{2}}=0
\end{aligned}
$$

The solution of the PDE can be derived using the method of characteristics

$$
\frac{\mathrm{d} z}{a r^{2}}=\frac{\mathrm{d} \varphi}{b}, \quad d \eta=0
$$

which leads to

$$
\begin{aligned}
& \eta=F(r, \varphi, z), \\
& C_{1}=-b z+a r^{2} \varphi .
\end{aligned}
$$

Finally, the coordinate $\eta$ reads

$$
\eta=F\left(C_{1}\right)=-b z+a r^{2} \varphi .
$$

We further show that, even though the coordinate lines of constant $\xi$ and $\eta$ are orthogonal, the three unit vectors $\boldsymbol{e}_{r}, \boldsymbol{e}_{\xi}$ and $\boldsymbol{e}_{\eta}$ are not orthogonal. 
The gradient operator in cylindrical coordinates is given by

$$
\nabla S=\frac{\partial S}{\partial r} \boldsymbol{e}_{r}+\frac{1}{r} \frac{\partial S}{\partial \varphi} \boldsymbol{e}_{\varphi}+\frac{\partial S}{\partial z} \boldsymbol{e}_{z}
$$

where $S$ is a scalar quantity. Using that, we obtain

$$
\boldsymbol{e}_{r}=\frac{\nabla r}{\|\nabla r\|}, \quad \boldsymbol{e}_{\xi}=\frac{\nabla \xi}{\|\nabla \xi\|}
$$

for the unit vectors $\boldsymbol{e}_{r}$ and $\boldsymbol{e}_{\xi}$. The gradient of the coordinate $\eta$ is given by

$$
\begin{aligned}
\nabla \eta & =\frac{\partial \eta}{\partial r} \boldsymbol{e}_{r}+\frac{1}{r} \frac{\partial \eta}{\partial \varphi} \boldsymbol{e}_{\varphi}+\frac{\partial \eta}{\partial z} \boldsymbol{e}_{z} \\
& =2 \operatorname{ar} \varphi \boldsymbol{e}_{r}+\operatorname{are}_{\varphi}-b \boldsymbol{e}_{z} .
\end{aligned}
$$

The absolute value reads

$$
\|\nabla \eta\|=r \sqrt{4 a^{2} \varphi^{2}+\frac{1}{B^{2}}}
$$

from which the unit vector of the invariant coordinate is determined:

$$
\boldsymbol{e}_{\eta}=\frac{\nabla \eta}{\|\nabla \eta\|}=\frac{1}{r \sqrt{4 a^{2} \varphi^{2}+\frac{1}{B^{2}}}}\left(\begin{array}{c}
2 \operatorname{ar} \varphi \\
a r \\
-b
\end{array}\right) .
$$

For orthogonality of the unit vectors, the condition that $\boldsymbol{e}_{r} \times \boldsymbol{e}_{\eta} \stackrel{!}{=} \boldsymbol{e}_{\xi}$ must hold. However, the cross product of both unit vectors is given by

$$
\begin{aligned}
\boldsymbol{e}_{r} \times \boldsymbol{e}_{\eta} & =\left(\begin{array}{l}
1 \\
0 \\
0
\end{array}\right) \times \frac{1}{r \sqrt{4 a^{2} \varphi^{2}+\frac{1}{B^{2}}}}\left(\begin{array}{c}
2 a r \varphi \\
a r \\
-b
\end{array}\right) \\
& =\frac{1}{r \sqrt{4 a^{2} \varphi^{2}+\frac{1}{B^{2}}}}\left(\begin{array}{c}
0 \\
b \\
a r
\end{array}\right) \neq \boldsymbol{e}_{\xi},
\end{aligned}
$$

which is only in the case of $\varphi=0$ equal to the unit vector $\boldsymbol{e}_{\xi}$.

\section{B Derivation of periodicity conditions for helical coordinates}

The transformation from helical to cylindrical coordinates is given by

$$
\left(\begin{array}{c}
r \varphi \\
z
\end{array}\right)=\left[\begin{array}{ll}
\boldsymbol{v} & \boldsymbol{w}
\end{array}\right]\left(\begin{array}{l}
\tilde{\zeta} \\
\eta
\end{array}\right)=\left[\begin{array}{ll}
v_{1} & w_{1} \\
v_{2} & w_{2}
\end{array}\right]\left(\begin{array}{l}
\tilde{\zeta} \\
\eta
\end{array}\right)
$$




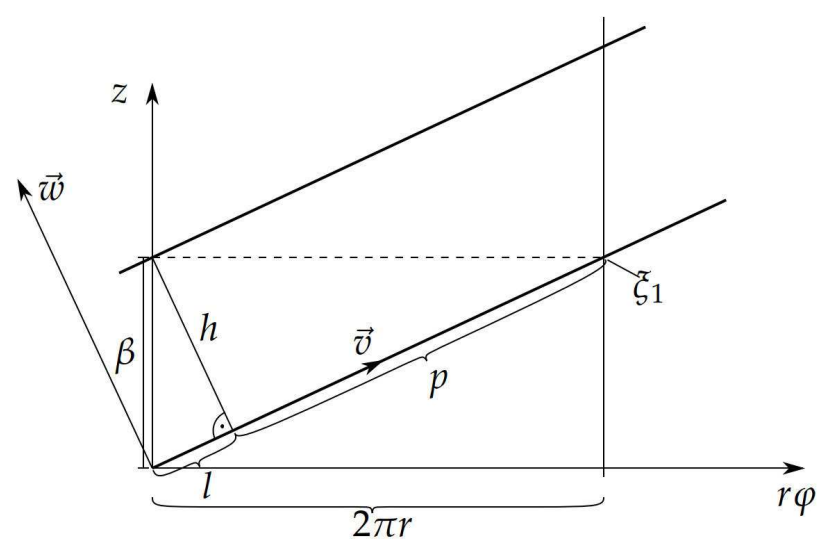

Figure 8: An illustration of the geometric correlations for one helical turn. The coordinate lines of constant $\xi$ are presented in the $r \varphi-z$-plane. $\beta$ is the pitch of the helix and $h$ the distance of two points on two helical turns.

and the inverse relation

$$
\left(\begin{array}{l}
\xi \\
\eta
\end{array}\right)=\underbrace{\left[\begin{array}{cc}
\frac{b}{r} & a \\
a r & -b
\end{array}\right]}_{M}\left(\begin{array}{c}
r \varphi \\
z
\end{array}\right)
$$

where

$$
\boldsymbol{M}^{-1}=\frac{1}{-\frac{b^{2}}{r}-a^{2} r}\left[\begin{array}{cc}
-b & -a \\
-a r & \frac{b}{r}
\end{array}\right] .
$$

That leads to

$$
\left(\begin{array}{c}
r \varphi \\
z
\end{array}\right)=\left[\begin{array}{cc}
\frac{b}{r} B^{2} & \frac{a}{r} B^{2} \\
a B^{2} & -\frac{b}{r^{2}} B^{2}
\end{array}\right]\left(\begin{array}{l}
\tilde{\zeta} \\
\eta
\end{array}\right)
$$

We now compute the point $\xi_{1}$ after one helical turn $\varphi=2 \pi$

$$
v_{1} \cdot \xi_{1}=2 \pi r
$$

which is given by

$$
\xi_{1}=\frac{2 \pi r^{2}}{b B^{2}} .
$$

Using that, the pitch of the helix reads

$$
\beta=v_{2} \cdot \xi_{1}=a B^{2} \cdot \frac{2 \pi r^{2}}{b B^{2}}=2 \frac{a}{b} \pi r^{2} .
$$

From Fig. 8, one may observe that

$$
h^{2}+p^{2}=4 \pi^{2} r^{2}
$$


and further, using $h^{2}=l \cdot p$, defining $x:=l+p$ and employing both into (B.7) leads to

$$
p=\frac{4 \pi^{2} r^{2}}{x}=2 \pi b B
$$

where we employed the trigonometric relation $x^{2}=\beta^{2}+4 \pi^{2} r^{2}$ and (B.6). From (B.8) it follows that for the case when $r \rightarrow 0$ the length $p$ vanishes, which means that there will be no $\xi$-dependence at the centerline. Similarly, knowing that $p=x-l$ we obtain

$$
l=\frac{\beta^{2}}{\sqrt{\beta^{2}+4 \pi^{2} r^{2}}} .
$$

Finally, we compute the periodic conditions $\xi_{p}, \xi_{l}, \eta_{p}$ considering the following relations of distances for one helical pitch

$$
\begin{aligned}
& p:(l+p)=\xi_{p}: \xi_{1}, \\
& l:(l+p)=\xi_{l}: \xi_{1}, \\
& \xi_{l} \cdot \boldsymbol{v}+\eta_{p} \cdot \boldsymbol{w}=\left(\begin{array}{c}
0 \\
\xi_{1} v_{2}
\end{array}\right),
\end{aligned}
$$

which are given by

$$
\begin{aligned}
& \xi_{p}=\frac{8 \pi^{3} r^{4}}{\left(\beta^{2}+4 \pi^{2} r^{2}\right) b B^{2}}=2 \pi b \\
& \xi_{l}=\frac{2 \pi \beta^{2} r^{2}}{\left(\beta^{2}+4 \pi^{2} r^{2}\right) b B^{2}}=\frac{2 \pi r^{2} a^{2}}{b}, \\
& \eta_{p}=-\frac{8 \pi^{3} r^{4}}{\left(\beta^{2}+4 \pi^{2} r^{2}\right) b B^{2}} \frac{a r^{2}}{b}=-2 \pi r^{2} a .
\end{aligned}
$$

\section{A condition for uniqueness at the centerline}

Expanding (5.1), we obtain

$$
\lim _{r \rightarrow 0} \frac{\partial u}{\partial \varphi}=\lim _{r \rightarrow 0}\left(\frac{\partial u^{r}}{\partial \varphi} \boldsymbol{e}_{r}+u^{r} \frac{\partial \boldsymbol{e}_{r}}{\partial \varphi}+\frac{\partial u^{\varphi}}{\partial \varphi} \boldsymbol{e}_{\varphi}+u^{\varphi} \frac{\partial \boldsymbol{e}_{\varphi}}{\partial \varphi}+\frac{\partial u^{z}}{\partial \varphi} \boldsymbol{e}_{z}+u^{z} \frac{\partial \boldsymbol{e}_{z}}{\partial \varphi}\right)
$$

The derivatives w.r.t. circumferential direction of the three cylindrical unit vectors are given by

$$
\frac{\partial \boldsymbol{e}_{z}}{\partial \varphi}=0, \quad \frac{\partial \boldsymbol{e}_{r}}{\partial \varphi}=\boldsymbol{e}_{\varphi}, \quad \frac{\partial \boldsymbol{e}_{\varphi}}{\partial \varphi}=-\boldsymbol{e}_{r}
$$

Hence, we obtain

$$
\lim _{r \rightarrow 0} \frac{\partial u}{\partial \varphi}=\lim _{r \rightarrow 0}\left(\left(\frac{\partial u^{r}}{\partial \varphi}-u^{\varphi}\right) \boldsymbol{e}_{r}+\left(\frac{\partial u^{\varphi}}{\partial \varphi}+u^{r}\right) \boldsymbol{e}_{\varphi}+\left(\frac{\partial u^{z}}{\partial \varphi}\right) \boldsymbol{e}_{z}\right)
$$


The components must vanish for $r \rightarrow 0$. This leads to

$$
\lim _{r \rightarrow 0}\left(\frac{\partial u^{r}}{\partial \varphi}-u^{\varphi}\right)=0, \quad \lim _{r \rightarrow 0}\left(\frac{\partial u^{\varphi}}{\partial \varphi}+u^{r}\right)=0, \quad \lim _{r \rightarrow 0}\left(\frac{\partial u^{z}}{\partial \varphi}\right)=0 .
$$

Next, we express the derived conditions (C.4) in helical coordinates derived in [28]. Employing the velocity components (2.7) and the derivative

$$
\frac{\partial}{\partial \varphi}=b \frac{\partial}{\partial \eta}+b \frac{\partial}{\partial \xi}
$$

Imposing helical invariance, i.e. $\left(\frac{\partial}{\partial \eta} \equiv 0\right)$, leads to

$$
\begin{aligned}
& \lim _{r \rightarrow 0}\left(b u_{\xi}^{r}-B\left(\frac{b}{r} u^{\xi}+a u^{\eta}\right)\right)=0, \\
& \lim _{r \rightarrow 0}\left(B \frac{b^{2}}{r} u_{\xi}^{\xi}-B b a u_{\xi}^{\eta}+u^{r}\right)=0, \\
& \lim _{r \rightarrow 0}\left(B b a u_{\xi}^{\xi}-\frac{b^{2}}{r} B u_{\xi}^{\eta}\right)=0 .
\end{aligned}
$$

$B(r)$ is the geometric function, for which $\lim _{r \rightarrow 0} B(r)=0$ hold. If we now assume

$$
\lim _{r \rightarrow 0} u^{r}< \pm \infty, \quad \lim _{r \rightarrow 0} \frac{\partial u^{\xi}}{\partial \xi}< \pm \infty, \quad \lim _{r \rightarrow 0} \frac{\partial u^{\eta}}{\partial \xi}< \pm \infty,
$$

i.e. $u^{r}$ is limited and $u^{\eta}$ and $u^{\xi}$ are smooth with respect to $\xi$ for $r \rightarrow 0$, we obtain

$$
\begin{aligned}
& \lim _{r \rightarrow 0}\left(b u_{\xi}^{r}-\frac{b}{\sqrt{a^{2} r^{2}+b^{2}}} u^{\xi}\right)=0, \\
& \lim _{r \rightarrow 0}\left(\frac{b^{2}}{\sqrt{a^{2} r^{2}+b^{2}}} u_{\xi}^{\xi}-u^{r}\right)=0, \\
& \lim _{r \rightarrow 0}\left(\frac{b^{2}}{\sqrt{a^{2} r^{2}+b^{2}}} u_{\xi}^{\eta}\right)=0 .
\end{aligned}
$$

Finally, the centerline conditions are given by

$$
b u_{\xi}^{r}=u^{\xi}, \quad b u_{\xi}^{\xi}=-u^{r}, \quad u_{\xi}^{\eta}=0
$$

for $r=0$. For the pressure, the condition is given by

$$
\lim _{r \rightarrow 0} \frac{\partial p}{\partial \varphi}=\frac{\partial p}{\partial \xi}=0 .
$$




\section{References}

[1] D. N. Arnold. An interior penalty finite element method with discontinuous elements. SIAM J. Numer. Anal., 19(4):742, 1982. ISSN 00361429.

[2] I. Babuška. The finite element method with Lagrangian multipliers. Numerische Mathematik, 20(3):179-192, 1973.

[3] E. Barbosa and O. Daube. A finite difference method for 3D incompressible flows in cylindrical coordinates. Computers E Fluids, 34(8):950-971, 2005.

[4] O. Bogoyavlenskij. Helically symmetric astrophysical jets. Phys. Rev. E, 62:8616-8627, Dec 2000a. doi: 10.1103/PhysRevE.62.8616. URL http://link.aps.org/doi/10.1103/ PhysRevE.62.8616.

[5] O. I. Bogoyavlenskij. Exact helically symmetric plasma equilibria. Letters in Mathematical Physics, 51(4):235-247, 2000b.

[6] O. I. Bogoyavlenskij. Helically symmetric astrophysical jets. Physical Review E, 62(6):8616, 2000c.

[7] S. L. Bragg and W. R. Hawthorne. Some exact solutions of the flow through annular cascade actuator discs. J. Aeronaut. Sci., 17:243-249, 1950.

[8] F. Brezzi and M. Fortin. Mixed and Hybrid Finite Element Methods, volume 15. Springer Science \& Business Media, 2012.

[9] J. Cheng, S. Yu, H. Yue, and T. Liu. An adjoint-based h-adaptive reconstructed discontinuous Galerkin method for the steady-state compressible Euler equations. Communications in Computational Physics, 26(3):855-879, 2019.

[10] A. Cheviakov and O. Bogoyavlenskij. Exact anisotropic mhd equilibria. Journal of Physics A: Mathematical and General, 37(30):7593, 2004. URL http://stacks . iop.org/0305-4470/37/ $i=30 / a=014$.

[11] G. S. Constantinescu and S. Lele. A highly accurate technique for the treatment of flow equations at the polar axis in cylindrical coordinates using series expansions. Journal of Computational Physics, 183(1):165-186, 2002.

[12] I. Delbende, M. Rossi, and O. Daube. DNS of flows with helical symmetry. Theoretical and Computational Fluid Dynamics, 26(1-4):141-160, 2012.

[13] D. A. Di Pietro and A. Ern. Mathematical Aspects of Discontinuous Galerkin Methods, volume 69. Springer Science \& Business Media, 2011.

[14] D. Dierkes. A high-order discontinuous Galerkin solver and exact solutions for helically invariant flows. PhD thesis, Technische Universität, Darmstadt, Juni 2020. URL http://tuprints. ulb.tu-darmstadt.de/11841/.

[15] D. Dierkes and M. Oberlack. Euler and Navier-Stokes equations in a new time-dependent helically symmetric system: Derivation of the fundamental system and new conservation laws. Journal of Fluid Mechanics, 818:344-365, 2017.

[16] D. Dritschel. Generalized helical Beltrami flows in hydrodynamics and magnetohydrodynamics. Journal of Fluid Mechanics, 222:525-541, 1991.

[17] T. Ellis, L. Demkowicz, and J. Chan. Locally conservative discontinuous Petrov-Galerkin finite elements for fluid problems. Computers \& Mathematics with Applications, 68(11):1530$1549,2014$.

[18] E. Ferrer and R. Willden. A high order discontinuous Galerkin finite element solver for the incompressible Navier-Stokes equations. Computers \& Fluids, 46(1):224-230, 2011.

[19] M. Germano. On the effect of torsion on a helical pipe flow. Journal of Fluid Mechanics, 125:1-8, 1982. doi: 10.1017/S0022112082003206. URL http://dx.doi.org/10.1017/ 
S0022112082003206.

[20] M. Germano. The dean equations extended to a helical pipe flow. Journal of Fluid Mechanics, 203:289-305, 1989. doi: 10.1017/S0022112089001473. URL http://dx.doi.org/10.1017/ S0022112089001473.

[21] V. Girault, B. Rivire, and M. F. Wheeler. A discontinuous Galerkin method with nonoverlapping domain decomposition for the Stokes and Navier-Stokes problems. Math. Comp., 74 (249):53-84, 2005a.

[22] V. Girault, B. Rivire, and M. F. Wheeler. A splitting method using discontinuous Galerkin for the transient incompressible Navier-Stokes equations. ESAIM: Mathematical Modelling and Numerical Analysis, 39(6):1115-1147, Nov. 2005b. ISSN 0764-583X, 1290-3841. doi: 10. 1051/m2an:2005048. URL http: //www.esaim-m2an.org/10.1051/m2an:2005048.

[23] H. Grad and H. Rubin. Hydromagnetic equilibria and force-free fields. Journal of Nuclear Energy, 7:284-285, 1958.

[24] M. D. Griffin, E. Jones, and J. D. Anderson Jr. A computational fluid dynamic technique valid at the centerline for non-axisymmetric problems in cylindrical coordinates. Journal of Computational Physics, 30(3):352-360, 1979.

[25] J. S. Hesthaven and T. Warburton. Nodal Discontinuous Galerkin Methods: Algorithms, Analysis, and Applications. Springer Science \& Business Media, 2007.

[26] M. Jamil and C. Fetecau. Helical flows of maxwell fluid between coaxial cylinders with given shear stresses on the boundary. Nonlinear Analysis: Real World Applications, 11(5):4302-4311, 2010.

[27] J. Johnson, C. Oberman, R. Kulsrud, and E. Frieman. Some stable hydromagnetic equilibria. 1(4):281-296, 1958. ISSN 00319171. doi: DOI:10.1063/1.1705886. URL http://dx .doi .org/ doi/10.1063/1.1705886.

[28] O. Kelbin, A. F. Cheviakov, and M. Oberlack. New conservation laws of helically symmetric, plane and rotationally symmetric viscous and inviscid flows. Journal of Fluid Mechanics, 721: 340-366, 2013.

[29] M. R. Khorrami, M. R. Malik, and R. L. Ash. Application of spectral collocation techniques to the stability of swirling flows. Journal of Computational Physics, 81(1):206-229, 1989.

[30] F. Kummer. Extended discontinuous Galerkin methods for two-phase flows: The spatial discretization. International Journal for Numerical Methods in Engineering, 109(2):259-289,2017.

[31] J. Lopez and J. Shen. An efficient spectral-projection method for the Navier-Stokes equations in cylindrical geometries: I. Axisymmetric cases. Journal of Computational Physics, 139(2):308326, 1998.

[32] A. Mitchell, S. Morton, and J. Forsythe. Wind turbine wake aerodynamics. Air Force Academy Colorado Springs, Dept. of Aeronautics, Report ADA425027, 1997.

[33] R. D. Nair, S. J. Thomas, and R. D. Loft. A discontinuous Galerkin global shallow water model. Monthly Weather Review, 133(4):876-888, 2005.

[34] T. Sarpkaya. On stationary and travelling vortex breakdowns. Journal of Fluid Mechanics, 45 (03):545-559, 1971.

[35] D. Schnack, E. Caramana, and R. Nebel. Three-dimensional magnetohydrodynamic studies of the reversed-field pinch. 28(1):321-333, 1985. ISSN 00319171. doi: DOI:10.1063/1.865151. URL http://dx.doi.org/doi/10.1063/1.865151.

[36] V. D. Shafranov. Propagation of an electromagnetic field in a medium with spatial dispersion. Soviet. Phys. JETP, 7:1019-1029, 1958.

[37] K. Shahbazi, P. F. Fischer, and C. R. Ethier. A high-order discontinuous Galerkin method for the unsteady incompressible Navier-Stokes equations. Journal of Computational Physics, 222 
(1):391-407, 2007.

[38] E. Tuttle. Laminar flow in twisted pipes. Journal of Fluid Mechanics, 219:545-570, 1990. doi: 10.1017/S002211209000307X. URL http://dx.doi.org/10.1017/S002211209000307X.

[39] L. Vermeer, J. Sorensen, and A. Crespo. Wind turbine wake aerodynamics. Progress in Aerospace Sciences, 39(67):467-510, 2003. ISSN 0376-0421. doi: 10.1016/ S0376-0421(03)00078-2. URL http://www.sciencedirect.com/science/article/pii/ S0376042103000782.

[40] R. Verzicco and P. Orlandi. A finite-difference scheme for three-dimensional incompressible flows in cylindrical coordinates. Journal of Computational Physics, 123(2):402-414, 1996.

[41] C. Wang. On the low-Reynolds-number flow in a helical pipe. Journal of Fluid Mechanics, 108:185-194, 1981. doi: 10.1017/S0022112081002073. URL http://dx.doi.org/10.1017/ S0022112081002073.

[42] K. Wang, J. Cheng, and T. Liu. High order finite difference scheme based on DG boundary treatment (FDBDG). Communications in Computational Physics, 25(5):1413-1445, 2019.

[43] L. Zabielski and A. Mestel. Steady flow in a helically symmetric pipe. Journal of Fluid Mechanics, 370:297-320, Sept. 1998.

[44] F. Zhang, J. Cheng, and T. Liu. A direct discontinuous Galerkin method for the incompressible Navier-Stokes equations on arbitrary grids. Journal of Computational Physics, 380: 269-294, 2019. 